\title{
Actively growing cells are the predominant persisters in exponential phase of Escherichia coli
}

\author{
Miki Umetani ${ }^{1,2,3 \ddagger}$, Miho Fujisawa ${ }^{3, \ddagger}$, Reiko Okura $^{3}$, Takashi Nozoe ${ }^{1,2,3}$, Shoichi \\ Suenaga $^{4}$, Hidenori Nakaoka ${ }^{5}$, Edo Kussell ${ }^{6,7}$, Yuichi Wakamoto ${ }^{1,2,3,{ }^{*}}$ \\ 1 Research Center for Complex System Biology, The University of Tokyo \\ 2 Universal Biology Institute, The University of Tokyo \\ 3 Department of Basic Science, Graduate School of Arts and Sciences, The \\ University of Tokyo \\ 4 Department of Neuropathology, Graduate School of Medicine, The \\ University of Tokyo \\ 5 Department of Gene Mechanisms, Graduate School of Biostudies, Kyoto \\ University \\ 6 Department of Biology, New York University \\ 7 Department of Physics, New York University \\ $\ddagger$ Equal contribution \\ * cwaka@mail.ecc.u-tokyo.ac.jp
}

\begin{abstract}
Bacterial persistence is a phenomenon in which a small fraction of isogenic bacterial cells survives a lethal dose of antibiotics. It is generally assumed that persistence is caused by growth-arrested dormant cells generated prior to drug exposure. However, evidence from direct observation is scarce due to extremely low frequencies of persisters, and is limited to high persistence mutants or to conditions that significantly increase persister frequencies. Here, utilizing a microfluidic device with a membrane-covered microchamber array, we visualize the responses of more than $10^{6}$ individual cells of wildtype Escherichia coli to lethal doses of antibiotics, sampling cells from different growth phases and culture media. We show that preexisting dormant persisters constitute only minor fractions of persistent cell lineages in populations sampled from exponential phase, and that most persistent cell lineages grew actively before drug exposure. Actively growing persisters exhibit radical morphological changes in response to drug exposure, including L-form-like morphologies or filamentation depending on antibiotic type, and restore their rod-like shape after drug removal. Incubating cells under stationary phase conditions increases both the frequency and the probability of survival of dormant cells. While dormant cells in late stationary phase express a general stress response regulator, RpoS, at high levels, persistent cell lineages tended to show low to moderate RpoS expression among the dormant cells. These results demonstrate that heterogeneous survival pathways may coexist within bacterial populations to achieve persistence and that persistence does not necessarily require dormant cells.
\end{abstract}

\section{Introduction}

Antibiotics are potent chemotherapeutic substances that are heavily relied upon to counter bacterial infectious diseases. Nonetheless, bacterial cells often circumvent 
elimination and continue to survive under antibiotics exposure by acquiring genetic changes to enhance their resistance or tolerance, or by producing less susceptible phenotypic variants. Bacterial persistence is a phenomenon in which a small subset of cells in an isogenic cell population survives for a prolonged period under exposure to lethal doses of antibiotics 118 . The phenomenon occurs across a wide range of combinations of bacterial species and antibiotics, including clinically important pathogens 8, , such as Mycobacterium tuberculosis [2, 9, 10, Staphylococcus aureus [1, 12], and Pseudomonas aeruginosa [13. Consequently, bacterial persistence has been recognized as a global health concern $1,4,0,9]$.

Since the first elaboration of persistence [14], growth-arrested dormant cells generated before drug exposure have been thought to be responsible for the persistence of cellular populations [1 8]. This explanation of persistence was based on the observations that most antibiotics were ineffective to bacterial cell populations under growth-inhibiting conditions, such as low temperature, low nutrients, and presence of bacteriostatic substances [14. Persistence caused by such prior exposure to stressful environments is now called triggered persistence 15]. If cells with inactive growth are present even in actively growing cellular populations, these cells are likely to survive antibiotic exposure for extended periods. However, examining this hypothesis by directly observing individual cell lineages is challenging due to extremely low frequencies of surviving cells (typically $10^{-6}-10^{-3}[16]$ ). Consequently, the evidence is limited to high persistence mutants 17, 18, to naturally high-persisting bacterial species 19, or to conditions that significantly increase the frequencies of surviving cells 20,21 . For example, Balaban et al. utilized high persistence (hip) mutants of Escherichia coli 22 and demonstrated that cells showing no or slow growth within actively growing populations survived ampicillin (Amp) exposure [17. Bakshi et al. performed time-lapse experiments with $E$. coli and applied Amp to cells regrowing from stationary phase [23], a known environmental condition that increases the frequency of persister cells 24]. They demonstrated that cells that remained dormant even after removing stationary phase conditions survived under subsequent Amp exposure 23].

Despite these experimental demonstrations, it still remains elusive whether any cellular traits other than dormancy could cause bacterial persistence. In fact, several studies have suggested that persistence occurs without dormant cells. For example, using a cell-sorting assay Orman and Brymildsen showed that even the fast-growing fraction of an exponentially growing $E$. coli population produced cells that survived lethal doses of Amp and ofloxacin 25]. Wakamoto et al. conducted single-cell observation of persistence of Mycobacterium smegmatis against isoniazid (INH), revealing that the surviving cells grew normally before drug exposure 19. These results imply that bacterial persistence occurs even without dormant cells. However, the survival of non-dormant cells is often deemed an exception in bacterial persistence 26.

To investigate whether dormant cells are indeed predominantly responsible for bacterial persistence, we visualized the response of individual cells to antibiotics exposure utilizing a microfluidic device equipped with a membrane-covered microchamber array (MCMA). This device allows us to directly observe the fates of more than $10^{5}$ individual bacterial cells. Using this device, we analyzed the persistence of wildtype E. coli (MG1655 strain), one of the best-studied model systems of bacterial persistence. Contrary to the prevailing view in the field, we reveal that most surviving cells were actively growing before drug exposure when Amp was exposed to the cell populations sampled from exponential phase. Non-dormant survivors exhibited radical morphological changes under Amp exposure and survived by transitioning to L-form-like cells. Cells that survived exposure to a fluoroquinolone antibiotic (ciprofloxacin, CPFX) were also actively growing cells. These results reveal that multiple survival pathways are available for persistence against the same drug depending on growth phases, and suggest 


\section{Results}

\section{The MCMA device unravels persistent single-cell lineages}

We constructed an E. coli strain MF1 that expresses RpoS-mCherry from the native rpoS locus on the chromosome. RpoS is a specialized sigma factor controlling the general stress response [27, and its high expression has been suggested to predict persistence 6]. This E. coli strain also expresses green fluorescent protein (GFP) from a low copy plasmid 28 to facilitate visualizing individual cells. The minimum inhibitory concentration (MIC) of MF1 against Amp was $16 \mu \mathrm{g} / \mathrm{ml}$, which was the same as that of its non-fluorescent parental strain MG1655 (Fig. S1A).

The population killing curve of MF1 sampled from exponential phase exhibited biphasic or multi-phasic decay in LB medium containing $200 \mathrm{\mu g} / \mathrm{ml}$ of Amp $(12.5 \times \mathrm{MIC}$, Fig. 1A; see Fig. $\mathrm{S} 2 \mathrm{~A}$ for the growth curve of this strain). Therefore, this E. coli strain exhibits persistence in this condition 15 . The decay rate markedly decreased under the Amp exposure from $0.18 \pm 0.02 \mathrm{~h}^{-1}$ to $0.005 \pm 0.003 \mathrm{~h}^{-1}$ (Fig. 11A). The frequency of persister cells evaluated at $3 \mathrm{~h}$ after the onset of Amp exposure was $4.2 \times 10^{-6}$ $\left(2.0 \times 10^{-6}-8.8 \times 10^{-6}\right)$ (Fig. 1 A) .

To visualize low-frequency persistent single-cell lineages, we used a microfluidic device equipped with a membrane-covered microchamber array (MCMA; Fig. 1B, 1 $\mathrm{C}$, and S3). E. coli cells were enclosed in the $0.8-\mu \mathrm{m}$ deep microchambers etched on a glass coverslip by covering microchambers with a cellulose membrane (Fig. 1 C). E. coli cells grow in a monolayer and form two-dimensional microcolonies in the microchambers (Fig. 1 C, 1D and S3). We can control medium conditions around cells flexibly by the medium flow above the membrane (Fig. $1 \mathrm{C}$ and $\mathrm{S} 3$ ). Here, we used the method of attaching cellulose membranes to glass coverslips via biotin-streptavidin binding, which was developed previously and utilized for analyzing bacterial growth and starvation [29 31].

We conducted time-lapse single-cell observation of MF1 cells using the MCMA device, sampling cells from exponential phase. We monitored 110-170 microchambers with a $100 \times$ objective in each time-lapse experiment to observe individual cells with a high spatial resolution. After initial pre-culturing periods of $1.5 \mathrm{~h}$ without Amp, we exposed cell populations to $200 \mathrm{\mu g} / \mathrm{ml}$ of Amp for $3.5 \mathrm{~h}$. The Amp exposure lysed most cells (Fig. 11D-I, Movie S1, and Movie S2). However, we detected two single-cell lineages that survived and regrew after the Amp exposure in the ten repeated experiments conducted with the MCMA device (Fig. 1D-I, Movie S1, and Movie S2). Interestingly, cells grew and divided actively before the Amp exposure in one of the persistent cell lineages (Fig. 1D-F and Movie S1). In response to the Amp exposure, the cell in this lineage radically changed its cell morphology from a rod shape to a round shape with membrane blebbing and transitted to an L-form-like cell 32 (Fig. 1 $1 \mathrm{E}$ and Movie S1). After drug removal, many progeny of the surviving cell gradually restored rod shapes through multiple cell divisions (Fig. 1D, 1 $1 \mathrm{E}$, and Movie S1).

The other surviving cell lineage exhibited no growth and division before and during the Amp exposure and restarted growth after drug removal (Fig. 1 G-I and Movie S2). This sequence of growth behavior is consistent with the expected survival dynamics for persistence caused by dormant cells [17].

Hereafter, we refer to the surviving cells that exhibited no growth and division before drug exposure (Fig. 1I) as dormant persisters and those that showed growth and division (Fig. 1F) as non-dormant persisters.

The expression levels of RpoS-mCherry were low in all the observed cells, including 

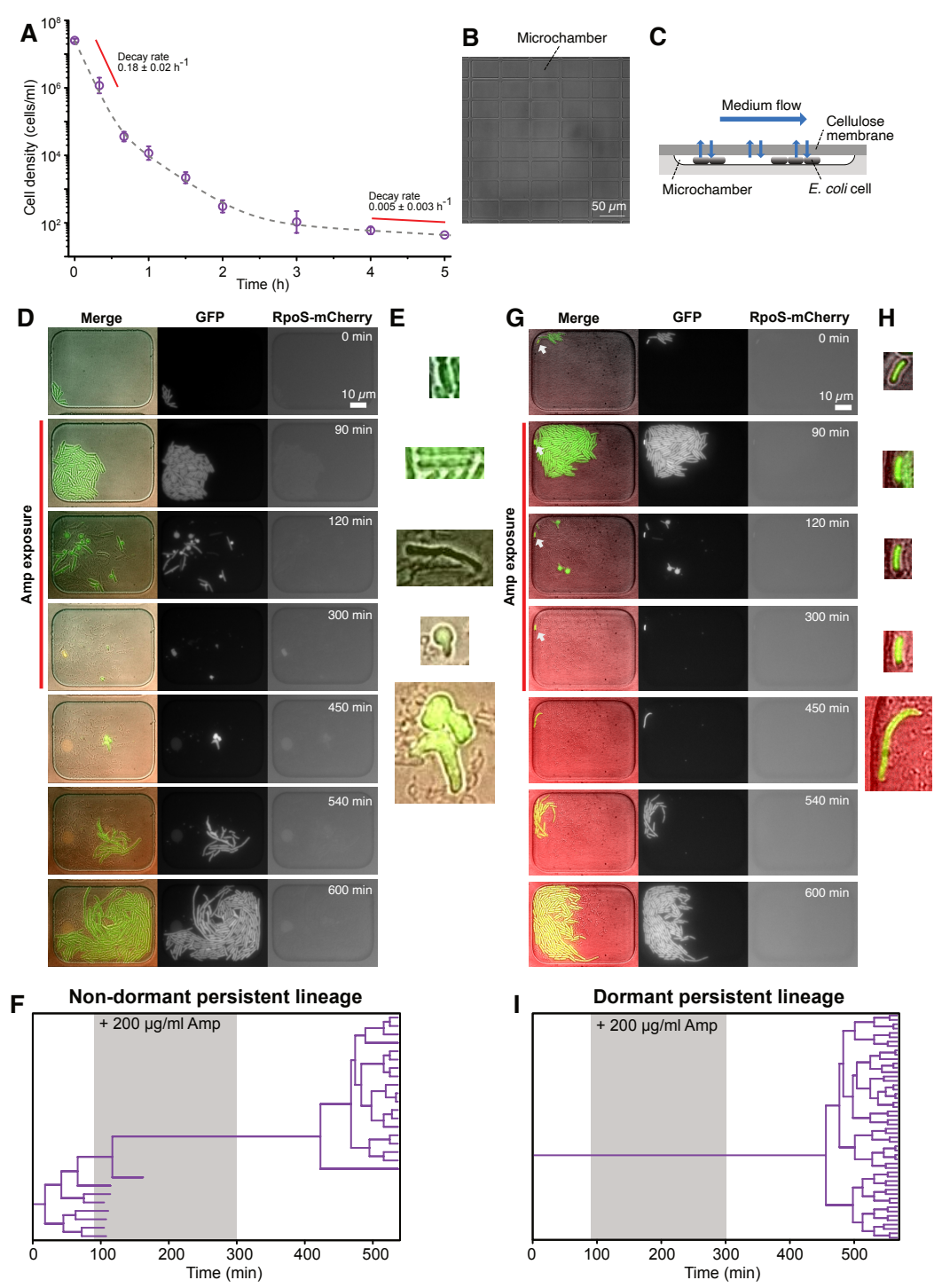

Figure 1. Detecting E. coli persistent cell lineages against Amp in exponential phase cultures. A. Population killing curve. E. coli MF1 cells in exponential phase were exposed to $200 \mathrm{\mu g} / \mathrm{ml}$ of Amp, and the viable cell density in the cell cultures at each time point was determined by a limiting dilution assay (see Materials and Methods). The points and the error bars represent the means and the standard errors of the logarithm of the cell density among three replicate experiments. The dashed curve represents the fitting of the triple exponential decay curve to the data: $N(t)=2.53 \times 10^{7} \exp (-0.183 t)+2.84 \times 10^{5} \exp \left(-5.73 \times 10^{-2} t\right)+1.89 \times 10^{2} \exp \left(-4.88 \times 10^{-3} t\right)$. B. A micrograph of the microchamber array etched in a glass coverslip. C. Schematic drawing of cell cultivation in the MCMA device. Cells are enclosed in the microchambers by a cellulose membrane cover. A culture medium flows above the membrane. The medium in the microchambers is exchanged through diffusion across the membrane. Cells grow in a monolayer until they are thoroughly packed in the microchambers. D. Time-lapse images of the microchamber with a non-dormant persister cell. The right and middle image sequences show the RpoS-mCherry and GFP fluorescence images. The left image sequence shows the merged images of bright-field (grayscale), GFP (green), and RpoS-mCherry (red) channels. The red line to the left of the images indicates the images under Amp exposure. E. Enlarged micrographs of the cells in the non-dormant persistent cell lineage in D. F. Single-cell lineage tree that produced the non-dormant persister. The lineage tree which originated from the ancestor cell of the non-dormant persister cell is shown. The gray background indicates the period of the Amp exposure. The bifurcations of the branches represent cell divisions. The endpoints of the branches under the Amp exposure represent cell lysis events. G. Time-lapse images of the microchamber with a dormant persister cell. The correspondence of the image sequences to the acquisition channels is the same as in D. The white arrows indicate the dormant cell that eventually proliferated after the Amp exposure. H. Enlarged micrographs of the cells in the dormant persistent cell lineage in G. I. Single-cell lineage tree that produced the dormant persister. 
the dormant persister cell characterized above (Fig. 1D, 1 G, Movie S1, and Movie S2). The suppression of RpoS expression in exponential phases in LB media is consistent with the literature 27,33 .

\section{Persisters are predominantly non-dormant in exponential-phase cell cultures exposed to Amp}

To further scale up single-cell observation, we next used a $40 \times$ objective and acquired only bright-field images in the time-lapse measurements (Movie S3). These changes in the microscopy configuration reduced the spatial resolution of acquired images and did not involve acquisition of fluorescence images. However, most single-cell lineages could still be tracked, and the drug response of more than $10^{5}$ individual cells was observable with a time-lapse interval of $3 \mathrm{~min}$.

We again observed the response of the MF1 cells sampled from exponential phase exposed to $200 \mu \mathrm{g} / \mathrm{ml} \mathrm{Amp} \mathrm{for} 3.5 \mathrm{~h}$, detecting 12 persistent cell lineages (defined as drug-exposed cells that survived Amp exposure and regrew after drug removal) among $3.0 \times 10^{5}$ drug-exposed cells (Movie S4-S6). We found that 10 out of 12 persistent cell lineages were non-dormant cells that grew and divided actively before the Amp exposure (Fig. 2A).

To test whether medium conditions influence persistence dynamics, as previously suggested 34, we repeated the experiments with M9 minimal medium. The killing curve of the E. coli MF1 cell populations sampled from exponential phase cultures in M9 minimal medium shows a biphasic curve, confirming the occurrence of persistence in this condition (Fig. S4] see Fig. $\mathrm{S} 2 \mathrm{~B}$ for the growth curve) and indicating a frequency of persister cells that was 4-fold higher than in LB (Fig. S4).

Single-cell time-lapse measurements detected 24 persistent cell lineages that regrew and divided after the exposure to $200 \mathrm{\mu g} / \mathrm{ml}$ of Amp for $6 \mathrm{~h}$ among $1.1 \times 10^{6}$

drug-exposed cells. Among them, we were able to determine the presence or absence of growth and division of 19 persistent cell lineages, of which 17 were growing and dividing before the Amp exposure (Fig. 2A and Movie S7-S9); the presence or absence of growth and division of 5 persistent cell lineages was indeterminate due to defocusing of cell images, crowding with neighboring cells, or translocation of cells within microchambers. Therefore, the dominance of non-dormant persisters in exponential phase was unchanged even in M9 medium.

Radical morphological changes of cell bodies were also observed in many non-dormant cells in M9 medium, including those in the persistent cell lineages. Surprisingly, some of the L-form-like cells crawled around like amoeba cells within the microchambers, detaching and leaving some parts of their cell bodies behind (Movie S10). Furthermore, rod-shaped regrowing cell populations emerged even from the small and left-behind cell bodies (Movie S10). These observations reveal the abilities of E. coli cells to sustain viability with abnormal morphologies and to restore rod shapes in the absence of agents that induced morphological changes.

\section{Stationary phase environments increase the frequencies of dormant persisters}

Several batch-culture assays have shown that stationary phase environments increase the frequencies of persister cells in bacterial cell populations $15,16,24$. We therefore sampled cells from early and late stationary phases in LB medium (Fig. S2A) and exposed them to $200 \mathrm{\mu g} / \mathrm{ml}$ of Amp in both batch and single-cell cultures.

As expected, the population killing curves showed significantly higher frequencies of surviving cells (Fig. 2B). The frequencies of surviving cells evaluated at the $3 \mathrm{~h}$ time 


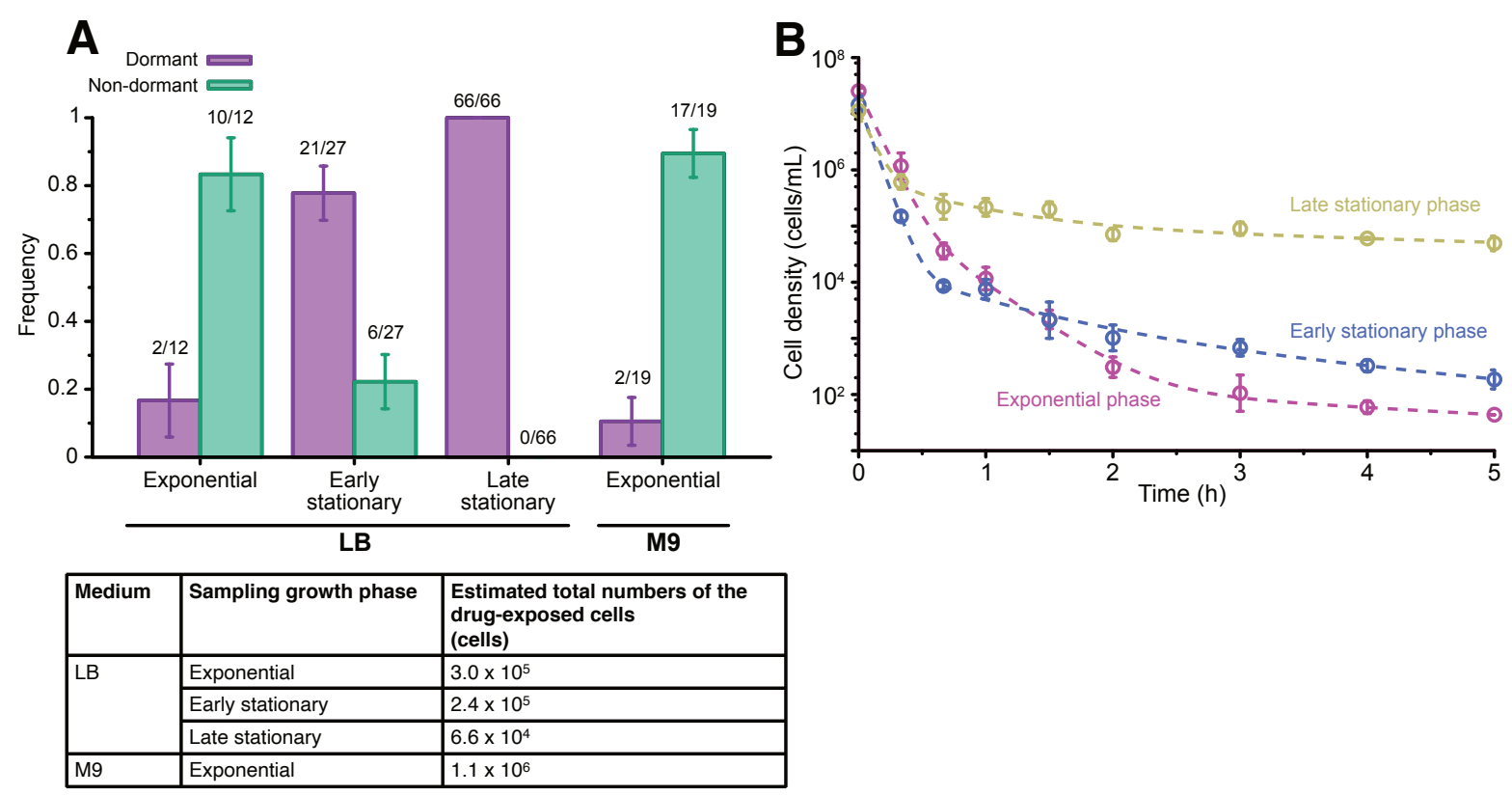

Figure 2. Growth-phase-dependent survival modes in the persistence against Amp. A. Proportions of dormant and non-dormant persisters. Purple and green bars represent the frequencies of dormant and non-dormant persisters among the persistent cell lineages detected in the time-lapse measurements of the MF1 cells. Error bars represent binomial standard errors. The number of cells categorized into each survival mode is shown above the bar alongside the total number of persistent cells in each culture condition for which the presence or absence of growth and division was distinguishable. The table shows the estimated total numbers of the drug-exposed cells observed in the time-lapse measurements. B. Killing curves of cell populations sampled from different growth phases. $200 \mathrm{\mu g} / \mathrm{ml}$ of Amp was applied to E. coli MF1 cell populations sampled from exponential phase (magenta), early stationary phase (blue), and late stationary phase (yellow). Points and error bars represent means and standard errors of the logarithm of cell density among three replicate experiments. The data of the exponential phase is the same as those shown in Fig. 17A. The dashed curves represent the fitting of the triple exponential decay curves to the data: $N(t)=1.50 \times 10^{7} \exp (-0.236 t)+1.89 \times 10^{5} \exp \left(-2.80 \times 10^{-2} t\right)+2.22 \times 10^{3} \exp \left(-8.29 \times 10^{-3} t\right)$ for the early stationary phase; $N(t)=1.07 \times 10^{7} \exp (-0.203 t)+5.41 \times 10^{5} \exp \left(-2.65 \times 10^{-2} t\right)+1.07 \times 10^{5} \exp \left(-2.45 \times 10^{-3} t\right)$ for the late stationary phase. 
point were $4.7 \times 10^{-5}\left(2.9 \times 10^{-5}-7.7 \times 10^{-5}\right)$ for the cell populations sampled from the early stationary phase and $7.9 \times 10^{-3}\left(4.8 \times 10^{-3}-1.3 \times 10^{-2}\right)$ for those from the late stationary phase (Fig. 2B).

Single-cell time-lapse measurements with the $40 \times$ objective detected 28 persistent cell lineages among $2.4 \times 10^{5}$ drug-exposed cells in populations sampled from early stationary phase (Movie S11-S13). Among them, we were able to determine growth states of 27 persistent cell lineages, of which 6 were non-dormant. However, the other 21 persistent cell lineages were dormant and exhibited no cell divisions before the Amp exposure (Fig. 2A). Therefore, the proportions of dormant persisters increased significantly in the cell populations sampled from early stationary phase.

For cell populations sampled from late stationary phase, we detected 69 persistent cell lineages among $6.6 \times 10^{4}$ drug-exposed cells, finding that all but 3 indeterminate lineages were dormant before the Amp exposure (Fig. 2A and Movie S14-S16). These results show that the predominance of different survival modes against Amp depends on the incubation duration under stationary phase conditions.

We found that many dormant cells sampled from late stationary phase remained intact under Amp exposure but did not regrow even after the drug removal. We confirmed that most of these were viable by propidium iodide (PI) staining (Fig. S5 and Movie S17). Hence, it is plausible that these cells would eventually regrow as persisters with long lags. In fact, it has been shown by the single-cell measurements with a mother machine microfluidic device that the time to regrowth after drug removal is heterogeneous among dormant cells 23. It should be noted that these late regrowers are detectable in the killing curve assay but not in the single-cell measurements with the MCMA microfluidic device due to the overgrowth of the early regrowers. Consequently, our single-cell measurements underestimate the frequency of persister cells in the population sampled from late stationary phase.

\section{Stationary phase environments enhance survivability of dormant cells against Amp exposure.}

To characterize the elongation and division dynamics of individual cells during the pre-culturing period in the MCMA device, we performed time-lapse experiments with cells sampled from early and late stationary phase using the $100 \times$ objective and observed lags in their regrowth (Fig. 3A-C, Movie S18 and S19). While most cells eventually restarted growth for populations sampled from early stationary phase, a significant fraction of cells remained non-growing and non-dividing during the pre-culture period of $2 \mathrm{~h}$ (Fig. 3B, C). The fractions of dormant cells in the cellular populations estimated by the elongation rates of individual cells immediately before the drug exposure were $(5.0 \pm 1.8) \times 10^{-3}(n=1612),(2.4 \pm 1.0) \times 10^{-2}(n=251)$, and $0.26 \pm 0.02$ $(n=818)$ in the cellular populations sampled from the exponential, early stationary, and late stationary phases, respectively (Fig. 3D-F). Therefore, the frequency of dormant cells in the populations increased significantly under stationary phase conditions.

We next asked whether the elevations of the fractions of persisters against Amp in the cellular populations sampled from the early and late stationary phases (Fig. $2 \mathrm{~B}$ ) were explainable by the increased fractions of dormant cells in the populations (Fig. 3). If the probability of dormant cells becoming persisters $\left(s_{d}\right)$ is independent of sampling growth phases and only dormant cells can survive, the frequency of surviving cells in the population $\left(f_{p}\right)$ estimated from the population killing curves should follow

$$
f_{p}=s_{d} P_{d}
$$

where $P_{d}$ is the fraction of dormant cells in the population. Since all the surviving cells in populations sampled from late stationary phase were dormant persisters (Fig. 2A), 

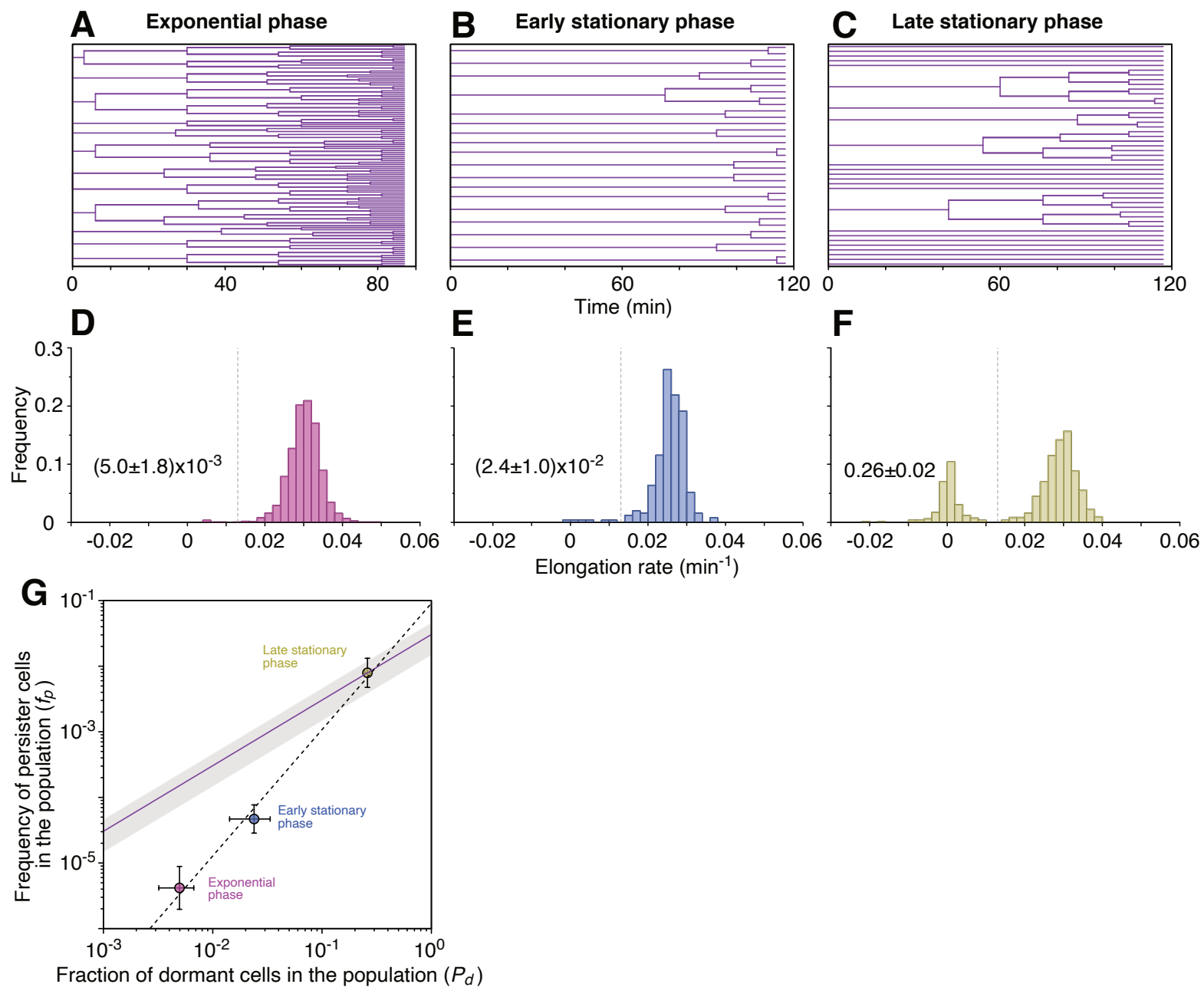

Figure 3. Stationary phase environments increase the frequency of dormant cells and enhance their survivability against Amp exposure. A-C. Representative single-cell lineage trees of cells sampled from exponential (A), early stationary (B), and late stationary (C) phases. In these trees, cells were cultured under the flow of fresh LB medium containing no drug. The cells sampled from early and late stationary phases had been maintained under growth-suppressive conditions under the flow of conditioned media prior to $t=0 \mathrm{~min}$ (see Materials and Methods). D-F. Elongation rate distributions before Amp exposure. We quantified the elongation rates of individual cells during the time window of 30 min immediately before the Amp exposure. D, E, and F are the distributions for the cell populations sampled from exponential, early stationary, and late stationary phases, respectively. The vertical dashed lines represent the threshold value of elongation rate $\left(0.013 \mathrm{~min}^{-1}\right)$ used to calculate the fractions of dormant cells shown in the plots. G. Relationship between the fractions of dormant cells $\left(P_{d}\right)$ and the frequency of surviving cells against Amp exposure $\left(f_{p}\right)$. The horizontal axis represents the fraction of dormant cells estimated in D-F. The vertical axis represents the frequency of surviving cells estimated from the frequencies of surviving cells in the population killing curves in Fig. $2 \mathrm{~B}$ at the time point of $3 \mathrm{~h}$ after the Amp exposure. The error bars along the horizontal axis represent the binomial standard errors, and those along the vertical axis represent the standard errors of the frequencies of the surviving cells. The purple line represents $f_{p}=s_{d} P_{d}$ when $s_{d}$ was assumed constant and estimated by the values of $f_{p}$ and $P_{d}$ for the late stationary phase condition. The gray background indicates the error ranges of $s_{d}\left(1.5 \times 10^{-2}-4.6 \times 10^{-2}\right)$. The dashed line represents the least-squares fitting of $f_{p}=e^{\alpha} P_{d}^{n}$ to the data in the log-log plot, and we found $\alpha=-2.4 \pm 0.6$ and $n=1.9 \pm 0.2$. 
we estimated $s_{d}$ as $f_{p}^{(\text {late })} / P_{d}^{(\text {late })}=(3.0 \pm 1.6) \times 10^{-2}$ from the frequencies of surviving cells $\left(f_{p}^{(\text {late })}\right)$ and of dormant cells $\left(P_{d}^{(\text {late })}\right)$ in populations from late stationary phase. Then, we predicted the frequencies of persister cells from the frequencies of dormant cells (Fig. 3D and E) in the populations sampled from exponential and early stationary phases.

The results revealed that the observed frequencies of surviving cells in the populations were significantly smaller than the frequencies predicted by Eq. 1 1 (Fig. $3 \mathrm{G}$ ). Note that the surviving cell frequencies under these conditions include the contributions of non-dormant persisters (Fig. 2A). Hence, $f_{p}$ should be greater than the prediction of Eq. 1 if $s_{d}$ were constant across the conditions. The result in Fig. $3 \mathrm{G}$ therefore indicates that the dormant cells in the population sampled from exponential and early stationary phases produced fewer surviving cells than expected from the frequencies of dormant cells. We conclude that long incubation under stationary phase conditions enhanced the survivability of dormant cells against the drug exposure.

\section{Growth phase dependent correlations between RpoS expression and persister trait}

We examined expression levels of RpoS in individual cells, finding that dormant cells in populations sampled from late stationary phase expressed RpoS at high levels at the onset of the Amp exposure (Fig. 44-F). Furthermore, we detected RpoS-mCherry foci in many dormant cells from late stationary phase (Fig. $4 \mathrm{G}$ and $\mathrm{H}$ ). This observation suggests that many of the RpoS-mCherry proteins were encapsulated into protein aggregates within the cells. This finding is consistent with previous results reported by [21] that long incubations under stationary phase conditions deepen dormancy depth and that the presence of protein aggregates characterizes the cells with deep dormancy.

Since dormant cells express RpoS at high levels and all the persisters emerge from the dormant cell fraction, high RpoS expression is necessarily found as the trait of persisters in the populations sampled from late stationary phase. However, dormant cells in populations sampled from exponential and early stationary phase did not necessarily express RpoS at high levels (Fig. 4 A A-F), and persister cells emerged even from the non-dormant cell fraction (Fig. 2A). Therefore, high RpoS expression cannot be a predictor of persister cells under these conditions.

Furthermore, in the populations sampled from late stationary phase, we noted that RpoS expression levels in persistent dormant lineages tended to be lower than those of all dormant cells (Fig. $4 \mathrm{~F}$, red points). The mean fluorescence intensity of RpoS-mCherry of the persistent dormant cells was $533 \pm 80$ a.u. $(n=8)$ immediately before the Amp exposure, whereas that of all the dormant cells was $758 \pm 19$ a.u. ( $n=213 ; p=0.034$, Welch's t-test). Therefore, high RpoS expression is not necessarily advantageous for dormant cells in terms of becoming persisters that can restart proliferation after drug removal.

\section{Actively growing cells produce persisters against ciprofloxacin in exponential phase cultures}

Having observed that the persisters against Amp in cellular populations sampled from exponential phase were predominantly actively-growing cells, we next investigated persistence against another antibiotic drug, ciprofloxacin (CPFX). While Amp is a $\beta$-lactam antibiotic targeting cell walls, CPFX is a fluoroquinolone antibiotic targeting DNA gyrase. MF1 cells in exponential phase in M9 minimal medium exposed to $1 \mathrm{\mu g} / \mathrm{ml}$ CPFX $(16 \times \mathrm{MIC}$, Fig. $\mathrm{S1} B)$ exhibited a biphasic killing curve, confirming the 

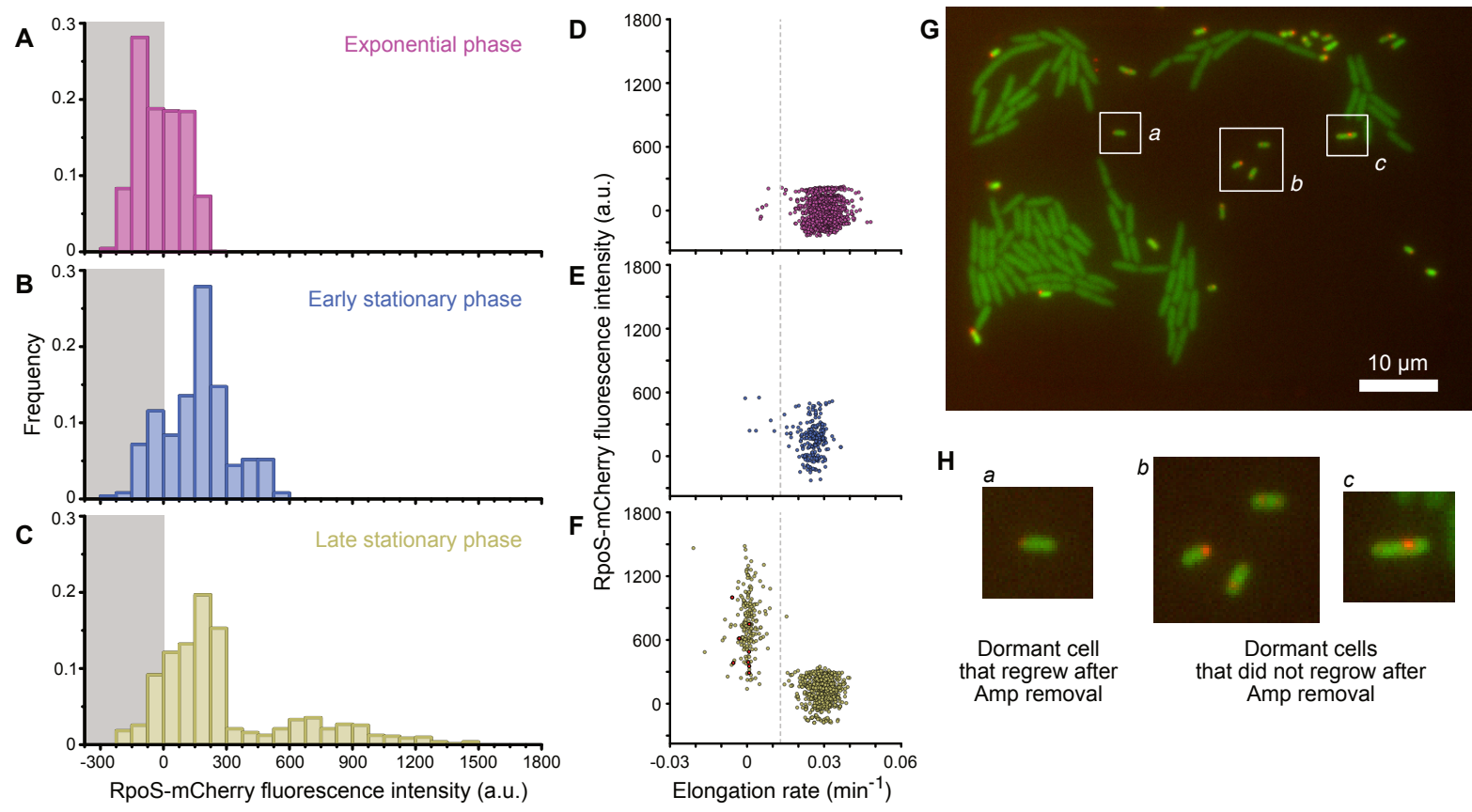

H

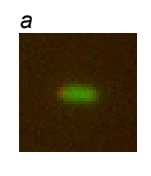

Dormant cell that regrew after Amp removal
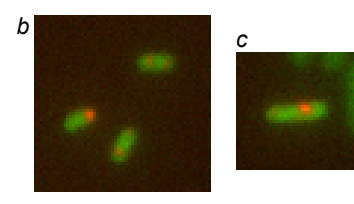

Dormant cells that did not regrow after Amp removal

Figure 4. Growth-phase-dependent expression levels of RpoS and its correlation with dormancy and persistence. A-C. Distributions of RpoS-mCherry fluorescence intensity at the onset of Amp exposure. We evaluated the mean fluorescence intensity of RpoS-mCherry (i.e., a proxy for the intracellular concentration) in individual cells at the onset of the Amp exposure. The cells were cultured in the microchambers from exponential (A), early stationary (B), and late stationary $(\mathrm{C})$ phases under the flow of fresh LB medium. The gray background indicates the intensity range in which the RpoS-mCherry fluorescence was indistinguishable from the background signals. D-F. Relationship between pre-exposure elongation rate and RpoS expression. The horizontal axis represents the elongation rates of individual cells immediately before the Amp exposure (see Fig. 3D-F for the distributions). The vertical axis represents the mean fluorescence intensity of individual cells at the onset of the Amp exposure. The vertical dashed lines represent the threshold elongation rate used to determine dormant cells. D, E, and F show the results for cells sampled from exponential, early stationary, and late stationary phases, respectively. The red points in F indicate cells that regrew after the Amp exposure of 3. 5 h. G. A representative micrograph of the MF1 cells sampled from late stationary phase. The merged image of GFP and RpoS-mCherry fluorescence channels at the onset of the Amp exposure is shown. H. Enlarged micrographs of the dormant cells in the image of G. $a, b$, and $c$ correspond to the respective squared frames in G. The cell in $a$ regrew after the removal of Amp. 
presence of persistence in this condition (Fig. S6). The frequency of persisters evaluated at the $3 \mathrm{~h}$ time point was $7.5 \times 10^{-4}\left(4.5 \times 10^{-4}-1.3 \times 10^{-3}\right)$.

We conducted time-lapse experiments using the MCMA device (Fig. 5 A, Movie S20, and Movie S21), and detected 148 persistent cell lineages that regrew after the exposure of $1 \mathrm{\mu g} / \mathrm{ml} \mathrm{CPFX}$ for $6 \mathrm{~h}$ among $8.3 \times 10^{5}$ drug-exposed cells. We found that cells grew and divided before the CPFX exposure in the detected persistent cell lineages (with 32 indeterminate lineages) (Fig. 5 . Movie S20, and Movie S21). Unlike Amp, the CPFX exposure did not lyse cells but arrested their growth and division, including those in the persistent cell lineages. After the removal of CPFX, the persister cells resumed growth and became filamentous. The filamentous cells restarted cell divisions and produced normal-sized, and normally growing and dividing, progeny cells. These results confirmed that persisters against CPFX in exponential phase were also non-dormant cells and thus persistence via non-dormant cells is not limited to Amp.

\section{Discussion}

Time-lapse single-cell observations with the MCMA microfluidic device enable direct visualization of persister cells existing at extremely low frequencies in isogenic bacterial cell populations. Contrary to the widely accepted view that pre-generated dormant cells are responsible for persistence, we found that actively growing cells produced persisters predominantly when the antibiotics were applied to the cellular populations sampled from exponential phases (Fig. 2A). Frequencies of dormant persisters increased in the cellular populations sampled from the early and late stationary phases (Fig. 2A). These results show that multiple survival modes are available for $E$. coli cells to achieve persistence even against the same drug. Furthermore, distinct survival modes coexisted within single persistent cell populations. In such cases, population-based analyses on bacterial persistence with an assumption of a single underlying mechanism would provide blurred results and bias our understanding of the phenomenon.

Interestingly, the predominant persisters against Amp in the cellular populations sampled from the exponential phases exhibited radical morphological changes and transitioned to the cell shapes that resemble L-form cells (Fig. 1 D and E). L-form bacteria are deficient in cell walls but can sustain growth and division under osmoprotective conditions 32,35 . L-form cells can be produced by treating bacteria with exogenous lytic enzymes or $\beta$-lactam antibiotics under osmoprotective conditions [35]. It is known that L-form bacteria are resistant to most antibiotics targeting cell wall synthesis, particularly $\beta$-lactams [32,35. Therefore, if the L-form-like cells produced by the Amp exposure could manage to protect themselves from osmotic pressure, they might be able to persist for an extended period under the Amp exposure. Since L-form cells are often found as antibiotic-resistant organisms and isolated from humans and animals in infectious diseases [35, this survival mode might be relevant to clinical settings. However, we also remark that such L-form-like cells did not appear upon CPFX exposure. Therefore, the transitions to L-form-like cells might be the general characteristics of non-dormant persisters against Amp and other $\beta$-lactam antibiotics, but these would not apply to the other non- $\beta$-lactam antibiotics.

In the time-lapse observations of the cells exposed to Amp, we found that some of the L-form-like cells crawled around in the microchambers. To the best of our knowledge, this is the first characterization that $E$. coli cells can exhibit such amoeboid migration. In eukaryotes, it has been shown that cells can migrate by blebbing in confined structures such as three-dimensional cultures and in vivo as an alternative to the lamellipodium-based motility [36]. Blebbing migration is driven by pressure rather than due to polymerizing actin filaments pushing against the membrane 36]. Therefore, a mechanism similar to blebbing migration may underlie the amoeboid migration 
A
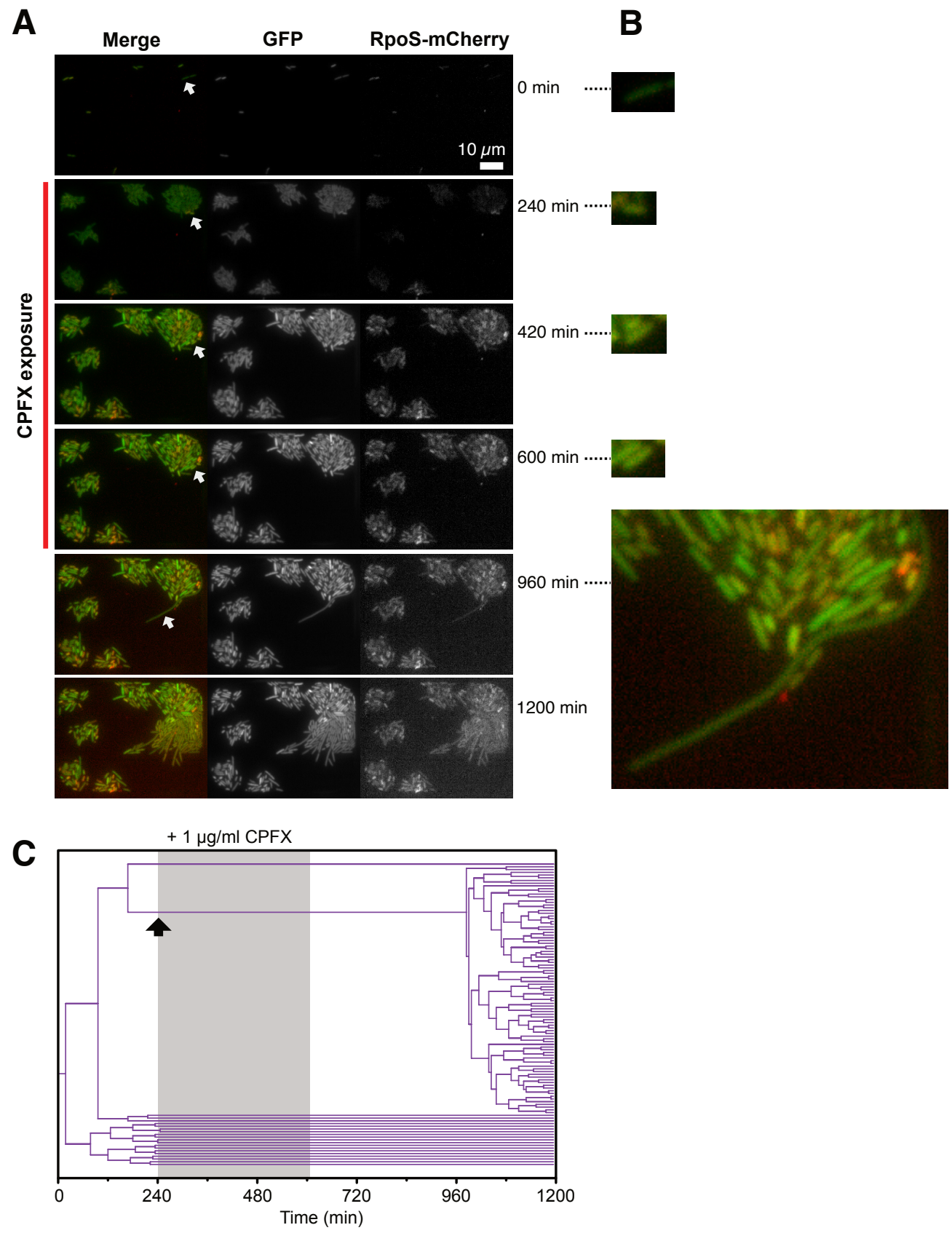

Figure 5. Detecting $\boldsymbol{E}$. coli persistent cell lineages against CPFX. A. Time-lapse images of the microchamber that produced a persister against CPFX. The right and middle image sequences show the RpoS-mCherry and GFP fluorescence images. The left image sequence shows the merged images of GFP (green) and RpoS-mCherry (red) channels. The white arrows indicate the cells in the persistent cell lineage. B. Enlarged micrographs of the cells in the persistent cell lineage. C. Single-cell lineage tree that produced the persister cell. Unlike lineage trees under Amp exposure, the CPFX exposure did not lyse the cells. The black arrow indicates the persister cell lineage that regrew after the 6 h CPFX exposure. 
observed in the L-form-like E. coli cells produced by the Amp exposure.

The result that the incubation under stationary phase conditions enhanced the survivability of dormant cells against the Amp exposure (Fig. $3 \mathrm{G}$ ) indicates that the states of dormant cells were not identical across the growth phases. In fact, many dormant cells in the populations sampled from the late stationary phase expressed RpoS at distinctly high levels in aggregated forms (Fig. $4 \mathrm{~F}-\mathrm{H})$. It is important to note that many dormant cells sampled from the late stationary phase remained viable under the Amp exposure (Fig S5 and Movie S17). Since it is reported that the time to regrowth of dormant cells after drug removal is heterogeneous among dormant cells [23, it is plausible that these cells would eventually regrow after long lags. However, these late regrowers cannot significantly contribute to the regrowing cell populations due to the exponential penalties from the delay of regrowth. It is also noteworthy that, in the cellular populations sampled from the late stationary phase, the RpoS expression levels of the dormant persisters that regrew after drug removal tended to be lower among the dormant cells (Fig. 4F). This observation may be consistent with a previous report that cells with shallow dormancy depth could restart proliferation quickly and become the main progenitors of the future population after drug removal [21].

As demonstrated here, our MCMA device can detect the persister cells existing at frequencies of $10^{-5}$ within a cellular population. This is the order at which one can detect persisters in most combinations of bacterial species and antibiotics, including clinically important ones $9,12,24$. Since multiple survival modes have been characterized in E. coli persistence, it would be crucial to unravel how individual cells of each bacterial species persist under various conditions of antibiotics exposure. Direct single-cell observations would unmask the diversity of persistence mechanisms and might enable rational designs of antibiotic treatments against bacterial infectious diseases.

\section{Materials and Methods}

\section{$E$. coli strains and culture conditions}

The main strain used in this study is E. coli strain MF1 (MG1655 rpoS-mcherry/pUA66- $\left.\mathrm{P}_{\mathrm{rplS}}-g f p\right)$. pUA66- $\mathrm{P}_{\mathrm{rplS}}-g f p$ is a low-copy plasmid taken from the E. coli promoter library 28]. In the propidium iodide (PI) staining experiment, we used MG1655/pUA66- $\mathrm{P}_{\mathrm{rplS}}-g f p$ since the PI emission spectrum overlaps with that of mCherry. We also used the MG1655 strain as a control in the MIC measurements.

To construct the MF1 strain, the mcherry gene was integrated downstream of the rpoS locus on the chromosome to express the RpoS-mCherry fusion protein from the native locus. We used the $\lambda$-Red recombination to integrate the mcherry gene [37.

We cultured the cells with either Luria-Bertani (LB) broth (Difco) or M9 minimal medium (Difco) supplemented with 1/2 MEM amino acids solution (SIGMA) and 0.2\% $(\mathrm{w} / \mathrm{v})$ glucose. All of the cultivation was conducted at $37^{\circ} \mathrm{C}$.

\section{Growth curve measurements}

$5 \mu \mathrm{L}$ of the glycerol stock cell suspension was inoculated into $2 \mathrm{~mL}$ of LB or M9 minimal medium containing $40 \mathrm{\mu g} / \mathrm{mL}$ of kanamycin (Kan), and we cultured the cells at $37^{\circ} \mathrm{C}$ for $15 \mathrm{~h}$ (LB) or $16 \mathrm{~h}$ (M9) with shaking. Kan was added to the culture media to avoid the loss of the GFP-expressing plasmid (pUA66- $\mathrm{P}_{\mathrm{rpls}}-g f p$ ) during the pre-culture. We diluted this pre-culture cell suspension with fresh LB or M9 medium (without Kan) to the cell density corresponding to the optical density at $600 \mathrm{~nm}\left(\mathrm{OD}_{600}\right)$ being 0.005 (LB) or 0.01 (M9). The total volume of the diluted cell cultures was adjusted to $50 \mathrm{~mL}$. After the dilution, we started culturing cells at $37^{\circ} \mathrm{C}$ with shaking. We sampled $100 \mu \mathrm{L}$ 
of the cell cultures every one hour and measured $\mathrm{OD}_{600}$ with a spectrophotometer (UV-1800, Shimadzu). We repeated the measurements three times. The mean and standard error of the logarithm of the $\mathrm{OD}_{600}$ were calculated for each sampling time point and plotted as the point and error bar in Fig. S2.

\section{Killing curve assay}

To measure killing curves in batch cultures, we first prepared $50 \mathrm{ml}$ cell suspensions of $\mathrm{OD}_{600}=0.005(\mathrm{LB})$ or $\mathrm{OD}_{600}=0.01$ (M9) following the same protocol of growth curve measurements. We incubated the diluted cell suspensions at $37^{\circ} \mathrm{C}$ with shaking and sampled a portion of the cell cultures at a pre-determined time point $(2.5 \mathrm{~h}$ for the exponential phase in LB; $8 \mathrm{~h}$ for the early stationary phase in LB; $24 \mathrm{~h}$ for the late stationary phase in LB; and $4 \mathrm{~h}$ for the exponential phase in M9). To match the pre-culturing conditions between the killing curve assays and the single-cell measurements, we diluted the extracted cell suspension with the fresh LB or M9 medium to the cell density with which the $\mathrm{OD}_{600}$ of the diluted cell suspension would reach 0.05 in the pre-culture of $2 \mathrm{~h}(\mathrm{LB})$ or $4 \mathrm{~h}$ (M9). The volume of the cell cultures was adjusted to $50 \mathrm{ml}$. After the pre-culture, we added the antibiotics (Amp or CPFX) to the cell culture and incubated it at $37^{\circ} \mathrm{C}$ with shaking. The concentrations of the antibiotics were adjusted to $200 \mu \mathrm{g} / \mathrm{ml}$ for Amp and $1 \mu \mathrm{g} / \mathrm{ml}$ for CPFX. We extracted $50 \mathrm{\mu l}$ or $500 \mathrm{\mu l}$ of the cell suspensions at the pre-determined time points to estimate the number of viable cells in the cellular populations.

To avoid the underestimation of viable cells from the non-culturability on solid agar 38, 39, we estimated the viable cell number by a limiting dilution method. We conducted serial dilution of the extracted cell suspension $(10 \times$ dilution in each dilution step) in $5 \mathrm{ml}$. We sampled $200 \mu \mathrm{l}$ of each diluted cell suspension into a well in a 96 -well plate. For each dilution condition, we dispensed the cell suspensions into 12 wells. We incubated the 96 -well plates at $37^{\circ} \mathrm{C}$ for $36 \mathrm{~h}$ (LB) or $48 \mathrm{~h}$ (M9), and the turbidity of each well was inspected.

We found one or two dilution conditions for each sample taken from different time points where both turbid and non-turbid wells coexist among the 12 wells. Assuming Poissonian allocation of viable cells, the proportion of non-turbid wells, $x_{0}$, is equal to $e^{-d y N_{v}}$, where $N_{v}$ is the original concentration of viable cells, $d$ is the dilution level, and $y(=0.2 \mathrm{ml})$ is the volume; then, $N_{v}=(y d)^{-1} \ln \left(1 / x_{0}\right)$. Thus, we estimated the concentration of viable cells in the cell population as $N_{v}=(5 / d) \ln (12 / k)$ cells $/ \mathrm{ml}$, where $k$ is the number of non-turbid wells. For example, if we found 3 non-turbid and 9 turbid wells for the cell suspension with the dilution of $10^{-6}$, we could estimate the viable cell density as $N_{v}=5 \times 10^{6} \times \ln (12 / 3)$ cells $/ \mathrm{ml}=7 \times 10^{6}$ cells $/ \mathrm{ml}$. When the coexistence of turbid and non-turbid wells was found in the two dilution conditions, we estimated the viable cell density of this time point by the geometric mean of the two estimated values.

We repeated the assay three times for each culture condition. The mean and standard error of the logarithm of the estimated viable cell density for each sampling time were calculated and shown as the point and error bar in Fig. 1 A, 2B, S4, and S6.

\section{MIC measurements}

$5 \mu \mathrm{l}$ of the glycerol stock of the $E$. coli strain was inoculated into $2 \mathrm{ml}$ of LB or M9 medium and incubated at $37^{\circ} \mathrm{C}$ with shaking for $15 \mathrm{~h}$ (LB) or $16 \mathrm{~h}$ (M9). We added Kan at the concentration of $40 \mu \mathrm{g} / \mathrm{ml}$ when we cultured the MF1 strain from the glycerol stock to avoid the loss of the GFP-expressing plasmid (pUA66-P $\mathrm{rpls}_{-}-g f p$ ) during the pre-culture. The cell cultures were diluted with a fresh LB or M9 medium to the cell density corresponding to $\mathrm{OD}_{600}=0.005(\mathrm{LB})$ or $\mathrm{OD}_{600}=0.01$ (M9). The diluted 
cell cultures of $2 \mathrm{ml}$ were incubated at $37^{\circ} \mathrm{C}$ with shaking for $2.5 \mathrm{~h}(\mathrm{LB})$ or $3 \mathrm{~h}(\mathrm{M} 9)$ to let the cell cultures enter exponential phases. The cell cultures were again diluted with a fresh medium to the cell density corresponding to $\mathrm{OD}_{600}=0.0002$. We mixed $100 \mu \mathrm{l}$ of the diluted cell suspension with $100 \mu \mathrm{l}$ of the medium containing Amp or CPFX and dispensed it into a well of the 96-well plate. We prepared 12 antibiotic concentration conditions with 2-fold serial differences (Amp: $2^{-2}-2^{8} \mu \mathrm{g} / \mathrm{ml}(+0 \mu \mathrm{g} / \mathrm{ml})$; CPFX: $\left.2^{-9}-2^{1} \mu \mathrm{g} / \mathrm{ml}(+0 \mu \mathrm{g} / \mathrm{ml})\right)$. We used three wells for each drug concentration condition. The cell cultures in the 96 -well plates were incubated at $37^{\circ} \mathrm{C}$ with shaking for $23 \mathrm{~h}$. We measured the optical density at $595 \mathrm{~nm}\left(\mathrm{OD}_{595}\right)$ of each well using a plate reader (FilterMax F5, Molecular Devices) and calculated the geometric mean of the three wells as the optical density of the cell culture in each drug concentration. We repeated the experiment three times and determined the MIC as the drug concentration above which the geometric mean of $\mathrm{OD}_{595}$ among the three replicate experiments were below 0.001 .

\section{Microfabrication}

We fabricated microchamber arrays on glass coverslips by wet etching. The dimensions of each microchamber were $62 \mu \mathrm{m}(\mathrm{w}) \times 47 \mu \mathrm{m}(\mathrm{h}) \times 0.8 \mu \mathrm{m}(\mathrm{d})$ for the array used in the experiments with a $100 \times$ objective (The number of microchambers in the array was 1240), and $53 \mu \mathrm{m}(\mathrm{w}) \times 33 \mu \mathrm{m}(\mathrm{h}) \times 0.8 \mu \mathrm{m}(\mathrm{d})$ for the array used in the experiments with a $40 \times$ objective (The number of microchambers in the array was 22,302 ).

We first made the photomask for the microchamber array by laser drawing (DDB-201-TW, Neoark) on mask blanks (CBL4006Du-AZP, CLEAN SURFACE TECHNOLOGY). The photoresist on mask blanks was developed in NMD-3 (Tokyo Ohka Kogyo). The uncovered chromium (Cr)-layer was removed in MPM-E30 (DNP Fine Chemicals), and the remaining photoresist was removed by acetone. Lastly, the slide was rinsed in MilliQ water and air-dried.

For creating microchambers based on the pattern on the photomask, we first coated a 1,000-angstrom Cr-layer on a clean coverslip (NEO Micro glass, No. 1., $24 \mathrm{~mm} \times 60$ $\mathrm{mm}$, Matsunami) by evaporative deposition and AZP1350 (AZ Electronic Materials) by spin-coating on the Cr-layer. After the pre-bake $\left(95^{\circ} \mathrm{C}, 90 \mathrm{sec}\right)$, we exposed the photoresist layer on the coverslip to UV light through the photomask using a mask aligner (MA-20, Mikasa). We developed the photoresist in NMD-3 and removed the Cr-layer uncovered by the photoresist in MPM-E30. The coverslip was soaked in buffered hydrofluoric acid solution (110-BHF, Morita Kagaku Kogyo) for 14 minutes 20 seconds at $23^{\circ} \mathrm{C}$ for glass etching. The etching reaction was stopped by soaking the coverslip in milliQ water. The remaining photoresist and the Cr-layer were removed by acetone and MPM-E30, respectively.

We used a polydimethylsiloxane (PDMS) pad with bubble trap groove to control and change the culture conditions around the cells in the microchamber array. We follow the same procedure as that reported in [40] to fabricate the PDMS pad.

\section{Chemical decoration of coverslip and cellulose membrane}

To enclose the $E$. coli cells in the microchambers, we employed a method of attaching a cellulose membrane to a glass coverslip via biotin-streptavidin binding [29]. We chemically decorated glass coverslips with biotin and cellulose membranes with streptavidin. We followed the same protocol reported in [40] to prepare biotin-decorated coverslips and streptavidin-decorated cellulose membranes. 


\section{Preparation of conditioned media}

As described below, we used the conditioned media to maintain the growth-suppressive conditions while preparing single-cell measurements of the cells sampled from the early and late stationary phases. We prepared $50 \mathrm{ml}$ of the $E$. coli cell suspension at $\mathrm{OD}_{600}$ $=0.005$ in the $\mathrm{LB}$ medium following the same procedure of growth curve measurements and incubated the cell cultures at $37^{\circ} \mathrm{C}$ with shaking. At the pre-determined time $(8 \mathrm{~h}$ for the early stationary phase condition and $24 \mathrm{~h}$ for the late stationary phase condition), we spun down the cells at $2,200 \times \mathrm{g}$ for $12 \mathrm{~min}$. The supernatant was again spun down at $2,200 \times \mathrm{g}$ for $12 \mathrm{~min}$, and its supernatant was filtered with a $0.2-\mu \mathrm{m}$ syringe filter. We used this filtered medium as the conditioned medium.

To prepare the agarose blocks of conditioned media, we dissolved $2 \%(\mathrm{w} / \mathrm{v})$ low-melt agarose in the conditioned media with a microwave and solidified it in a plastic dish. To use this for attaching a cellulose membrane to a coverslip, we excised a piece of the conditioned medium pad with a sterilized blade and placed it on the cellulose membrane.

\section{Sample preparation for time-lapse measurements}

To prepare $E$. coli cells for the time-lapse single-cell observation, $5 \mu \mathrm{l}$ of the glycerol stock of the MF1 cells was inoculated into $2 \mathrm{~mL}$ LB or M9 medium and incubated at $37^{\circ} \mathrm{C}$ with shaking for $15 \mathrm{~h}$. The cell culture was diluted to $\mathrm{OD}_{600}=0.005$ (in the case of $\mathrm{LB}$ medium) or $\mathrm{OD}_{600}=0.01$ (in the case of $\mathrm{M} 9$ medium) in a $50 \mathrm{~mL}$ fresh medium and again incubated at $37^{\circ} \mathrm{C}$ with shaking. We sampled cells from the cell culture at the pre-determined time points $(2.5 \mathrm{~h}$ for the exponential phase in LB; $8 \mathrm{~h}$ for the early stationary phase in LB; $24 \mathrm{~h}$ for the late stationary phase in LB; and $4 \mathrm{~h}$ for the exponential phase in M9).

For the exponential phase samples (both LB and M9), $1 \mu \mathrm{L}$ of cell suspension was sampled from the culture and placed on the microchamber array. We put a streptavidin-decorated cellulose membrane on the cell suspension and removed an excess suspension with a clean filter paper. A small piece of an LB or M9 agarose block ( $2 \%$ $(\mathrm{w} / \mathrm{v})$ agarose) was placed on the cellulose membrane and incubated at $37^{\circ} \mathrm{C}$ for $15 \mathrm{~min}$ to allow the membrane to adhere to the coverslip surface tightly. After removing the agarose block, a PDMS pad was attached to the coverslip via a Frame-Seal Incubation Chamber $(9 \mathrm{~mm} \times 9 \mathrm{~mm}$ or $15 \mathrm{~mm} \times 15 \mathrm{~mm}$, Bio-Rad). We connected the medium inlet and outlet of the PDMS pad to silicone tubes and started flowing an LB or M9 medium immediately at the flow rate of $2 \mathrm{~mL} / \mathrm{h}$ using syringe pumps (NE-1000, New Era Pump Systems). We then registered the microchamber positions and started the time-lapse measurements as described in the following section.

For the early and late stationary phase samples, we followed the same protocol except that we used the conditioned medium agarose pads made from the cell cultures of the corresponding growth phases to attach the cellulose membrane. We also flowed the liquid conditioned medium while registering the microchamber positions to maintain the growth-suppressive conditions. We switched the flowing media from the conditioned media to the fresh LB medium at the start of the time-lapse measurements to arrange the same duration of regrowth before the Amp exposure across the replicate experiments.

\section{Time-lapse observation with the MCMA microfluidic device}

We used Nikon Ti-E microscopes equipped with ORCA-flash camera (Hamamatsu Photonics) for the time-lapse observations. For the time-lapse observations with a $40 \times$ objective (Plan Apo $\lambda, 40 \times / \mathrm{NA} 0.95$ ), we acquired only bright-field images to monitor many microchamber positions simultaneously. For the time-lapse observations with a 
$100 \times$ objective (Plan Apo $\lambda, 100 \times /$ NA1.45, oil immersion), we acquired bright-field, RpoS-mCherry fluorescence, and GFP fluorescence images. We used the LED (MCWHL2, Thorlabs) for fluorescence excitation. We maintained the temperature around the microscope stage at $37^{\circ} \mathrm{C}$ with a thermostat chamber (TIZHB, Tokai Hit).

The microscope was controlled from a PC using Micromanager [41]. The time-lapse intervals were $3 \mathrm{~min}$ in the measurements with the LB medium and 6 min in the measurements with the M9 medium.

In the time-lapse measurements of the cells in the LB medium, we first allowed the cells to grow in the microchambers flowing a fresh medium for either $1.5 \mathrm{~h}$ (the cells from the exponential phase) or $2 \mathrm{~h}$ (the cells from the early and late stationary phases). Then, we changed the flowing media to the one containing $200 \mathrm{\mu g} / \mathrm{mL}$ of Amp and observed the cells flowing drug-containing medium for $3.5 \mathrm{~h}$. We changed the flowing media again to the fresh medium containing no drug and allowed the viable cells to regrow.

In the time-lapse measurements of the cells in the M9 medium, we allowed the cells to grow in the microchambers for $4 \mathrm{~h}$ flowing a fresh M9 medium. We then switched the flowing media to the one containing $200 \mathrm{\mu g} / \mathrm{ml}$ Amp or $1 \mathrm{\mu g} / \mathrm{ml}$ CPFX and observed the cells under the drug-exposed conditions for $6 \mathrm{~h}$. We again switched the flowing media back to the M9 medium containing no drug and allowed the viable cells to regrow.

\section{Image analysis}

We used a custom ImageJ macro to analyze the time-lapse images acquired with the $100 \times$ objective. This macro finds the outlines of the cells in each image and assigns the cell lineages based on the segmented cell image sequences. We corrected the errors in the segmentation and lineage assignments manually. The cell size (area), mean fluorescence intensity, and the connections in the cell lineages were exported into a text file for the data analysis.

We opened the images as a stack to find the persister cell lineages in the time-lapse images acquired with the $40 \times$ objective. From the end time point, we played back the stacked images to detect the microchambers with the surviving cells that stably regrew after the drug removal. We tracked back the persistent cell lineages and determined the existence of growth and cell divisions before the drug exposure.

To estimate the number of drug-exposed cells, we randomly selected 20 microchambers and counted the number of cells in each microchamber. We multiplied the averaged number of the cells in a microchamber by the number of the microchambers tracked in the time-lapse measurement. We used this number as the estimate of the total number of the drug-exposed cells in each experiment.

\section{Data analysis}

Estimation of persister frequencies We estimated the persister cell frequencies from the population killing curves as the number of surviving cells at the time point of 3 $\mathrm{h}$ under the drug exposure relative to the initial number of cells at the onset of drug exposure. The error ranges of the persister cell frequencies were estimated by the error propagation of the standard errors of the numbers of cells at the two time points.

Estimation of killing rates from the killing curves We estimated the killing rates of the cell populations by fitting the population killing curves. We conducted non-linear fitting of double exponential (M9) or triple exponential decay (LB) curves to the logarithms of the experimental data using the Levenberg-Marquardt algorithm on Gnuplot. We used triple exponential decay curves to fit the killing curves in the LB medium because of the significant fitting deviations by the double exponential decay 
curves. In the main text, we reported the rates of the fastest and slowest decay terms as the killing rates of the initial and persistence phases, respectively. The standard errors of the decay rates estimated by fitting were reported as their error ranges.

Estimation of dormant cell fractions in cellular populations We analyzed the time-lapse data using a custom $\mathrm{C}$ script and exported the data for plotting the cell lineage trees. To determine the elongation rates of individual cells before the Amp exposure, we focused on the time window of 90 min immediately before the Amp exposure and calculated the averages of the instantaneous elongation rate $\frac{1}{\Delta t} \ln \frac{S(t+\Delta t)}{S(t)}$ over this time window, where $\Delta t=3 \mathrm{~min}$ is the time-lapse interval and $S(t)$ is the cell size (area) at time $t$. We did not include the time points of cell divisions for calculating the averages if cells divided in this time window. We regarded cell lineages with elongation rates below $0.013 \mathrm{~min}^{-1}$ as dormant and calculated the frequency of such lineages in each cell population sampled from a different growth phase.

\section{Viability check by PI staining}

We found that many dormant cells in the populations sampled from the late stationary phase remained intact during the Amp exposure but did not regrow after the drug removal. We examined the viability of the dormant cells flowing the media containing $1 \mathrm{\mu g} / \mathrm{ml}$ PI in the time-lapse measurements. In these experiments, we used the MG1655 pUA66- $\mathrm{P}_{\mathrm{rplS}}-g f p$ strain since the emission spectrum of RpoS-mCherry overlaps with that of PI. The preparation of the cells for the time-lapse measurements was the same as that used for the cells sampled from the late stationary phase, except that we flowed the media containing PI from the time point of adding Amp.

\section{Acknowledgments}

This work was supported by JST CREST Grant Number JPMJCR1927 (Y.W.); JST ERATO Grant Number JPMJER1902 (Y.W.); NIH Grant Number R01-GM097356 (E.K.); and Japan Society for the Promotion of Science KAKENHI Grant Number 17 H06389 and 19H03216 (Y.W.).

\section{References}

1. Levin BR, Rozen DE. Non-inherited antibiotic resistance. Nature Reviews Microbiology. 2006;4(7):556-62. doi:10.1038/nrmicro1445.

2. Dhar N, McKinney JD. Microbial phenotypic heterogeneity and antibiotic tolerance. Current Opinion in Microbiology. 2007;10(1):30-8. doi:10.1016/j.mib.2006.12.007.

3. Lewis K. Persister cells, dormancy and infectious disease. Nature Reviews Microbiology. 2007;5(1):48-56. doi:10.1038/nrmicro1557.

4. Jayaraman R. Bacterial persistence: some new insights into an old phenomenon. Journal of Biosciences. 2008;33(5):795-805.

5. Balaban NQ. Persistence: mechanisms for triggering and enhancing phenotypic variability. Current Opinion in Genetics \& Development. 2011;21(6):768-75. doi:10.1016/j.gde.2011.10.001. 
6. Maisonneuve E, Gerdes K. Molecular mechanisms underlying bacterial persisters. Cell. 2014;157(3):539-548. doi:10.1016/j.cell.2014.02.050.

7. Kester JC, Fortune SM. Persisters and beyond: mechanisms of phenotypic drug resistance and drug tolerance in bacteria. Critical Reviews in Biochemistry and Molecular Biology. 2014;49(2):91-101. doi:10.3109/10409238.2013.869543.

8. van den Bergh B, Fauvart M, Michiels J. Formation, physiology, ecology, evolution and clinical importance of bacterial persisters. FEMS Microbiology Reviews. 2017;41(3):219-251. doi:10.1093/femsre/fux001.

9. Gomez JE, McKinney JD. M. tuberculosis persistence, latency, and drug tolerance. Tuberculosis. 2004;84(1-2):29-44. doi:10.1016/j.tube.2003.08.003.

10. Keren I, Minami S, Rubin E, Lewis K. Characterization and Transcriptome Analysis of Mycobacterium tuberculosis Persisters. mBio. 2011;2(3):e00100-11. doi:10.1128/mBio.00100-11.

11. Lechner S, Lewis K, Bertram R. Staphylococcus aureus persisters tolerant to bactericidal antibiotics. Journal of Molecular Microbiology and Biotechnology. 2012;22(4):235-44. doi:10.1159/000342449.

12. Johnson PJT, Levin BR. Pharmacodynamics, Population Dynamics, and the Evolution of Persistence in Staphylococcus aureus. PLoS Genet. 2013;9(1):e1003123. doi:10.1371/journal.pgen.1003123.

13. Liebens V, Defraine V, Van der Leyden A, De Groote VN, Fierro C, Beullens S, et al. A putative de-N-acetylase of the PIG-L superfamily affects fluoroquinolone tolerance in Pseudomonas aeruginosa. Pathogens and disease. 2014;71(1):39-54. doi:10.1111/2049-632X.12174.

14. Bigger JW. Treatment of staphylococcal infections with penicillin by intermittent sterilisation. The Lancet. 1944;244(6320):497-500.

15. Balaban NQ, Helaine S, Lewis K, Ackermann M, Aldridge B, Andersson DI, et al. Definitions and guidelines for research on antibiotic persistence. Nature Reviews Microbiology. 2019;17:441-448. doi:10.1038/s41579-019-0196-3.

16. Keren I, Kaldalu N, Spoering A, Wang Y, Lewis K. Persister cells and tolerance to antimicrobials. FEMS Microbiology Letters. 2004;230(1):13-18. doi:10.1016/S0378-1097(03)00856-5.

17. Balaban NQ, Merrin J, Chait R, Kowalik L, Leibler S. Bacterial persistence as a phenotypic switch. Science. 2004;305(5690):1622-5. doi:10.1126/science.1099390.

18. Svenningsen MS, Veress A, Harms A, Mitarai N, Semsey S. Birth and Resuscitation of (p)ppGpp Induced Antibiotic Tolerant Persister Cells. Scientific Reports. 2019;9:1-13. doi:10.1038/s41598-019-42403-7.

19. Wakamoto Y, Dhar N, Chait R, Schneider K, Signorino-Gelo F, Leibler S, et al. Dynamic persistence of antibiotic-stressed mycobacteria. Science. 2013;339:91-5. doi:10.1126/science.1229858.

20. Bamford RA, Smith A, Metz J, Glover G, Titball RW, Pagliara S. Investigating the physiology of viable but non-culturable bacteria by microfluidics and time-lapse microscopy. BMC Biology. 2017;15(1):1-12. doi:10.1186/s12915-017-0465-4. 
21. Pu Y, Li Y, Jin X, Tian T, Ma Q, Zhao Z, et al. ATP-Dependent Dynamic Protein Aggregation Regulates Bacterial Dormancy Depth Critical for Antibiotic Tolerance. Molecular Cell. 2019;73(1):143-156.e4. doi:10.1016/j.molcel.2018.10.022.

22. Moyed HS, Bertrand KP. hipA, a newly recognized gene of Escherichia coli K-12 that affects frequency of persistence after inhibition of murein synthesis. Journal of bacteriology. 1983;155:768-75.

23. Bakshi S, Leoncini E, Baker C, Cañas-Duarte SJ, Okumus B, Paulsson J. Tracking bacterial lineages in complex and dynamic environments with applications to growth control and persistence. bioRxiv. 2021; p. 2020.03.27.006403. doi:10.1101/2020.03.27.006403.

24. Spoering AL, Lewis K. Biofilms and planktonic cells of Pseudomonas aeruginosa have similar resistance to killing by antimicrobials. Journal of Bacteriology. 2001;183:6746-6751. doi:10.1128/JB.183.23.6746-6751.2001.

25. Orman MA, Brynildsen MP. Dormancy is not necessary or sufficient for bacterial persistence. Antimicrobial Agents and Chemotherapy. 2013;57(7):3230-3239. doi:10.1128/AAC.00243-13.

26. Balaban NQ, Gerdes K, Lewis K, McKinney JD. A problem of persistence: still more questions than answers? Nature Reviews Microbiology. 2013;11(8):587-91. doi:10.1038/nrmicro3076.

27. Battesti A, Majdalani N, Gottesman S. The RpoS-mediated general stress response in Escherichia coli. Ann Rev Microbiol. 2011;34:189-213.

28. Zaslaver A, Bren A, Ronen M, Itzkovitz S, Kikoin I, Shavit S, et al. A comprehensive library of fluorescent transcriptional reporters for Escherichia coli. Nature methods. 2006;3:623-8. doi:10.1038/nmeth895.

29. Inoue I, Wakamoto Y, Moriguchi H, Okano K, Yasuda K. On-chip culture system for observation of isolated individual cells. Lab on a Chip. 2001;1(1):50-5. doi:10.1039/b103931h.

30. Umehara S, Wakamoto Y, Inoue I, Yasuda K. On-chip single-cell microcultivation assay for monitoring environmental effects on isolated cells. Biochemical and Biophysical Research Communications. 2003;305. doi:10.1016/S0006-291X(03)00794-0.

31. Shimaya T, Okura R, Wakamoto Y, Takeuchi KA. Scale invariance during bacterial reductive division observed by an extensive microperfusion system. arXiv:200404903. 2021;doi:10.1101/2020.06.25.171710.

32. Errington J, Mickiewicz K, Kawai Y, Wu LJ. L-form bacteria, chronic diseases and the origins of life. Philosophical Transactions of the Royal Society B: Biological Sciences. 2016;371. doi:10.1098/RSTB.2015.0494.

33. Jishage M, Ishihama A. Regulation of RNA polymerase sigma subunit synthesis in Escherichia coli: intracellular levels of sigma 70 and sigma 38. Journal of Bacteriology. 1995;177(23):6832-5.

34. Harms A, Fino C, Sørensen MA, Semsey S, Gerdes K. Prophages and Growth Dynamics Confound Experimental Results with Antibiotic-Tolerant Persister Cells. mBio. 2017;8(6):1-18. doi:10.1128/mBio.01964-17. 
35. Allan EJ, Hoischen C, Gumpert J. Bacterial L-Forms. Advances in Applied Microbiology. 2009;68:1-39. doi:10.1016/S0065-2164(09)01201-5.

36. Charras G, Paluch E. Blebs lead the way: how to migrate without lamellipodia. Nature Reviews Molecular Cell Biology. 2008;9:730-736. doi:10.1038/nrm2453.

37. Datsenko KA, Wanner BL. One-step inactivation of chromosomal genes in Escherichia coli K-12 using PCR products. Proceedings of the National Academy of Sciences of the United States of America. 2000;97:6640-5. doi:10.1073/pnas.120163297.

38. Gelman E, McKinney JD, Dhar N. Malachite green interferes with postantibiotic recovery of mycobacteria. Antimicrobial Agents and Chemotherapy. 2012;56:3610-3614. doi:10.1128/AAC.00406-12.

39. Nosho K, Fukushima H, Asai T, Nishio M, Takamaru R, Kobayashi-Kirschvink $\mathrm{KJ}$, et al. cAMP-CRP acts as a key regulator for the viable but non-culturable state in Escherichia coli. Microbiology. 2018; p. 1-10. doi:10.1099/mic.0.000618.

40. Yamauchi S, Nozoe T, Okura R, Kussell E, Wakamoto Y. A unified framework for measuring selection on cellular lineages and traits. bioRxiv. 2021;doi:10.1101/2021.07.18.452747.

41. Edelstein AD, Tsuchida MA, Amodaj N, Pinkard H, Vale RD, Stuurman N. Advanced methods of microscope control using $\mu$ Manager software. Journal of Biological Methods. 2014;1:e11. doi:10.14440/jbm.2014.36. 


\section{Supplementary Information}

\section{Supplementary Movies}

Movie S1. Time-lapse observation of the non-dormant persister of the MF1 strain against Amp. The time-lapse images were acquired with a $100 \times$ objective. Left: Merged images of the bright-field (grayscale), GFP (green), and RpoS-mCheerry (red) channels; Middle: GFP channel; Right: RpoS-mCherry channel. The medium conditions are indicated near the upper-left corner. The counter near the lower-right corner indicates the time in hour:min. Scale bar, $10 \mu \mathrm{m}$.

Movie S2. Time-lapse observation of the dormant persister of the MF1 strain against Amp. The time-lapse images were acquired with a $100 \times$ objective. Left: Merged images of the bright-field (grayscale), GFP (green), and RpoS-mCheerry (red) channels; Middle: GFP channel; Right: RpoS-mCherry channel. The medium conditions are indicated near the upper-left corner. The counter near the lower-right corner indicates the time in hour:min. Scale bar, $10 \mu \mathrm{m}$.

Movie S3. Simultaneous time-lapse observation of the drug responses of many individual cells using the MCMA microfluidic device. This movie shows a single field of view observed in a time-lapse measurement with the MCMA microfluidic device using a $40 \times$ objective. 40 microchambers can be visualized per position. We imaged 50-150 positions in a single time-lapse measurement. In this movie, one microchamber harbored a persistent cell lineage against Amp exposure (the third row from the top and the second column from the left). The medium conditions are indicated near the upper-left corner. The counter near the lower-left corner indicates the time in hour:min. Scale bar, $50 \mu \mathrm{m}$.

Movie S4. Time-lapse movie of the MF1 persisters against Amp in the LB medium (Exponential phase, Replicate experiment 1). This movie shows all the microchambers harboring persistent cell lineages in one of the replicated single-cell measurements of the MF1 cells against Amp cultured in the LB medium. The cells were sampled from the exponential phase. We used a $40 \times$ objective in this measurement. Scale bar, $10 \mu \mathrm{m}$.

Movie S5. Time-lapse movie of the MF1 persisters against Amp in the LB medium (Exponential phase, Replicate experiment 2). See the caption of Movie S4 for detail.

Movie S6. Time-lapse movie of the MF1 persisters against Amp in the LB medium (Exponential phase, Replicate experiment 3). See the caption of Movie S4 for detail.

Movie S7. Time-lapse movie of the MF1 persisters against Amp in the M9 medium (Exponential phase, Replicate experiment 1). This movie shows all the microchambers harboring persistent cell lineages in one of the replicated single-cell measurements of the MF1 cells against Amp cultured in the M9 medium. The cells were sampled from the exponential phase. We used a $40 \times$ objective in this measurement. Scale bar, $10 \mu \mathrm{m}$. 
Movie S8. Time-lapse movie of the MF1 persisters against Amp in the M9 medium (Exponential phase, Replicate experiment 2). See the caption of Movie S7 for detail.

Movie S9. Time-lapse movie of the MF1 persisters against Amp in the M9 medium (Exponential phase, Replicate experiment 3). See the caption of Movie S7 for detail.

Movie S10. Amoeba-like locomotion of L-form-like $E$. coli cells in the microchambers. This movie shows representative three microchambers in which L-form-like E. coli cells exhibited amoeba-like locomotion. In this experiment, $200 \mathrm{\mu g} / \mathrm{ml}$ of Amp was exposed to the MF1 E. coli cells cultured in the M9 medium. Fission of cell bodies occurred in these cells during the locomotion. Furthermore, regrowing cell populations emerged from left-behind cell bodies.

Movie S11. Time-lapse movie of the MF1 persisters against Amp in the LB medium (Early stationary phase, Replicate experiment 1). This movie shows all the microchambers harboring persistent cell lineages in one of the replicated single-cell measurements of the MF1 cells against Amp cultured in the LB medium. The cells were sampled from the early stationary phase. We used a $40 \times$ objective in this measurement. Scale bar, $10 \mu \mathrm{m}$.

Movie S12. Time-lapse movie of the MF1 persisters against Amp in the LB medium (Early stationary phase, Replicate experiment 2). See the caption of Movie S11 for detail.

Movie S13. Time-lapse movie of the MF1 persisters against Amp in the LB medium (Early stationary phase, Replicate experiment 3). See the caption of Movie S11 for detail.

Movie S14. Time-lapse movie of the MF1 persisters against Amp in the LB medium (Late stationary phase, Replicate experiment 1). This movie shows all the microchambers harboring persistent cell lineages in one of the replicated single-cell measurements of the MF1 cells against Amp cultured in the LB medium. The cells were sampled from the late stationary phase. We used a $40 \times$ objective in this measurement. Scale bar, $10 \mu \mathrm{m}$.

Movie S15. Time-lapse movie of the MF1 persisters against Amp in the LB medium (Late stationary phase, Replicate experiment 2). See the caption of Movie S14 for detail.

Movie S16. Time-lapse movie of the MF1 persisters against Amp in the LB medium (Late stationary phase, Replicate experiment 3 ). See the caption of Movie S14 for detail.

Movie S17. Viability check of the non-regrowing dormant cells by PI staining. This movie shows the time-lapse observation of the

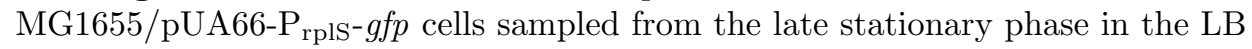
medium. We used a $100 \times$ objective in this measurement. Left: Merged images of the bright-field (grayscale), GFP (green), and PI (red) channels; Middle: GFP channel; Right: PI channel. PI was added to the flowing media from the start of the Amp 
exposure, as indicated by the medium conditions near the upper-left corner. We found many dormant cells that did not regrow even after the removal of Amp. They were visible in the GFP channel but negative with the PI staining. The counter near the upper-right corner indicates the time in hour:min. Scale bar, $10 \mu \mathrm{m}$.

Movie S18. Time-lapse observation of the MF1 cells sampled from the early stationary phase. The time-lapse images were acquired with a $100 \times$ objective. The cells were sampled from the early stationary phase in the LB medium and monitored in the microchambers. $200 \mathrm{\mu g} / \mathrm{ml}$ of Amp was exposed to the cells from $2 \mathrm{~h}$. The elongation rates and the RpoS-mCherry expression levels of individual cells before the Amp exposure were analyzed in Fig. 3 and 4 . Left: Merged images of the bright-field (grayscale), GFP (green), and RpoS-mCheerry (red) channels; Middle: GFP channel; Right: RpoS-mCherry channel. The medium conditions are indicated near the upper-left corner. The counter near the lower-right corner indicates the time in hour:min. Scale bar, $10 \mu \mathrm{m}$.

Movie S19. Time-lapse observation of the MF1 cells sampled from the late stationary phase. The time-lapse images were acquired with a $100 \times$ objective. The cells were sampled from the late stationary phase in the LB medium and monitored in the microchambers. $200 \mathrm{\mu g} / \mathrm{ml}$ of Amp was exposed to the cells from $2 \mathrm{~h}$. The elongation rates and the RpoS-mCherry expression levels of individual cells before the Amp exposure were analyzed in Fig. 3 and 4 . Left: Merged images of the bright-field (grayscale), GFP (green), and RpoS-mCheerry (red) channels; Middle: GFP channel; Right: RpoS-mCherry channel. The medium conditions are indicated near the upper-left corner. The counter near the lower-right corner indicates the time in hour:min. Scale bar, 10 m.

Movie S20. Time-lapse observation of the persister cells against CPFX (with $100 \times$ objective). The MF1 cells sampled from the exponential phase in the M9 medium were observed with the $100 \times$ objective. Left: Merged images of the GFP (green) and RpoS-mCherry (red) channels; Middle: GFP channel; Right:

RpoS-mCherry channel. $1 \mathrm{\mu g} / \mathrm{ml}$ of CPFX was exposed to the cells from $4 \mathrm{~h}$. The medium conditions are indicated near the lower-left corner. The counter near the lower-right corner indicates the time in hour:min. Scale bar, $10 \mu \mathrm{m}$.

Movie S21. Time-lapse observation of the persister cells against CPFX (with $40 \times$ objective). This movie shows all the microchambers harboring persistent cell lineages in one of the replicated single-cell measurements of the MF1 cells against CPFX cultured in the LB medium. The cells were sampled from the exponential phase. We used a $40 \times$ objective in this measurement. Scale bar, $10 \mu \mathrm{m}$. 


\section{Supplementary Figures}

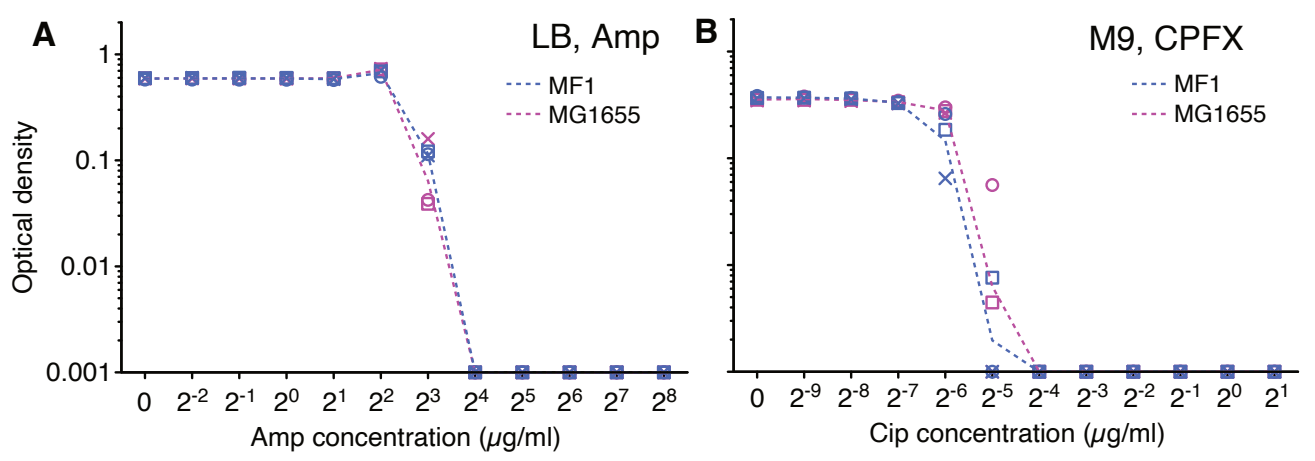

Figure S1. MIC tests. A. MIC test against Amp. The optical density at $595 \mathrm{~nm}$ of the $E$. coli cell cultures after the incubation of $23 \mathrm{~h}$ at $37^{\circ} \mathrm{C}$ in the $\mathrm{LB}$ media containing different concentrations of Amp (horizontal axis) is shown. Blue is for the MF1 strain, and magenta is for the MG1655 strain. The points (circles, squares, and crosses) represent the results of three replicate experiments. The dashed lines connect the geometric means of the replicates. The optical density values below 0.001 were plotted as 0.001 as they were indistinguishable from the fluctuation of background signals. B. MIC test against CPFX. The color correspondences to the E. coli strains are the same as that in A. The cells were incubated in the M9 media containing different concentrations of CPFX (horizontal axis) in this test. We measured the optical density at $595 \mathrm{~nm}$ after the incubation of $23 \mathrm{~h}$ at $37^{\circ} \mathrm{C}$. 


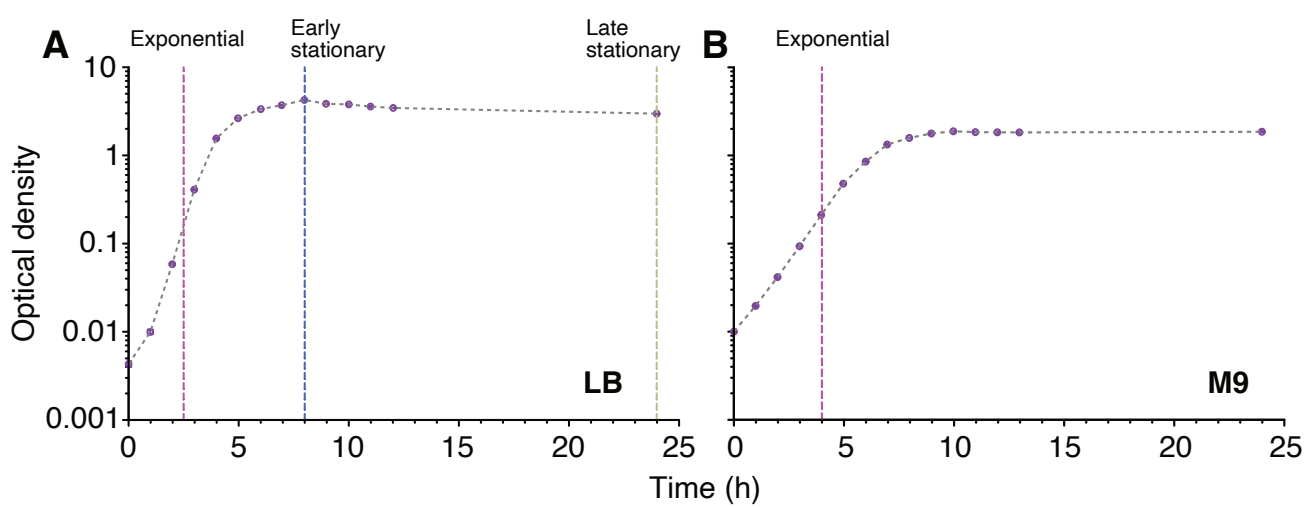

Figure S2. Population growth curves of the MF1 strain. A. The growth curve in the LB medium. The transitions of the optical density at $600 \mathrm{~nm}$ of the MF1 cell cultures are shown. The points represent the geometric means of three replicate experiments. The error bars represent the standard errors of the logarithm of the optical density, which are smaller than the point size. The vertical dashed lines represent the time points at which we sampled the cell cultures for the single-cell measurements and the killing curve assay. The magenta, blue, and yellow dashed lines indicate the sampling time points for the exponential, early stationary, and late stationary phases, respectively. B. The growth curve in the M9 medium. The points and the error bars (smaller than the point size) represent the same as those in A. The dashed line represents the time when cells were sampled for the single-cell measurements and killing curve assay. 


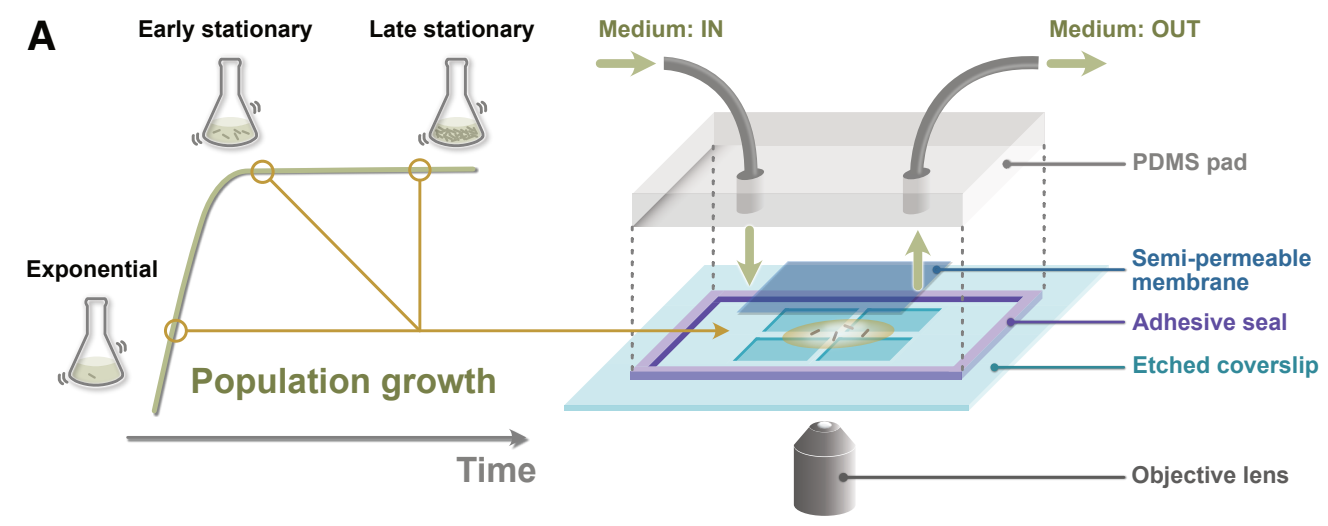

B

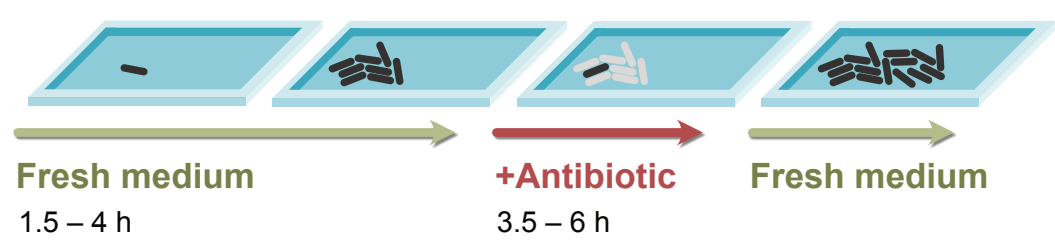

Figure S3. Experimental procedures for single-cell time-lapse measurements. A. Loading E. coli cells in the MCMA device. A suspension of E. coli cells sampled from a batch culture is placed on the microchamber array etched in a coverslip. Placing a semi-permeable membrane on the cell suspension randomly seeds the cells in the microchambers. The semi-permeable membrane tightly seals the microchambers via the biotin-streptavidin binding. A PDMS pad attached to the coverslip via an adhesive frame seal chamber allows medium perfusion. The scale of the microchambers is exaggerated in this diagram for an intuitive explanation. B. Sequence of environmental conditions in the time-lapse measurements. E. coli cells were first grown under the flow of fresh medium. After that, we flowed a medium containing an antibiotic (Amp or CPFX) and allowed the surviving cells to regrow by exchanging the media back to the fresh medium. 


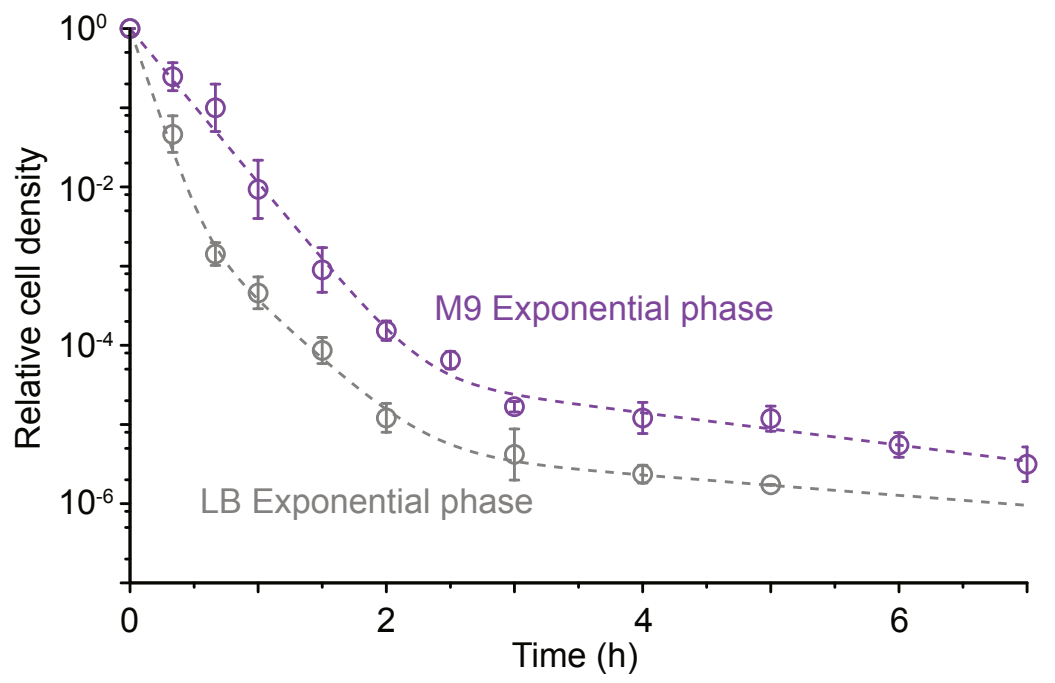

Figure S4. Comparison of the killing curves of the MF1 cells against Amp between LB and M9 media. $200 \mathrm{\mu g} / \mathrm{ml}$ of Amp was exposed to the MF1 cell cultures in the exponential phases in LB (gray) and M9 (purple) media. The points represent the geometric means of the relative cell density to the values at the onset of the Amp exposure (0 min) among three replicate experiments. Error bars represent the standard errors of the logarithm of the relative cell density. The data for the LB medium are the same as those in Fig. 1A except that they are shown as the relative values in this plot. The purple dashed curve represents the fitting of the double exponential decay curve to the data for the cells in the M9 medium: $N(t)=$ $\left(1-9.22 \times 10^{-5}\right) \exp \left(-7.47 \times 10^{-2} t\right)+9.22 \times 10^{-5} \exp \left(-7.83 \times 10^{-3} t\right)$. 


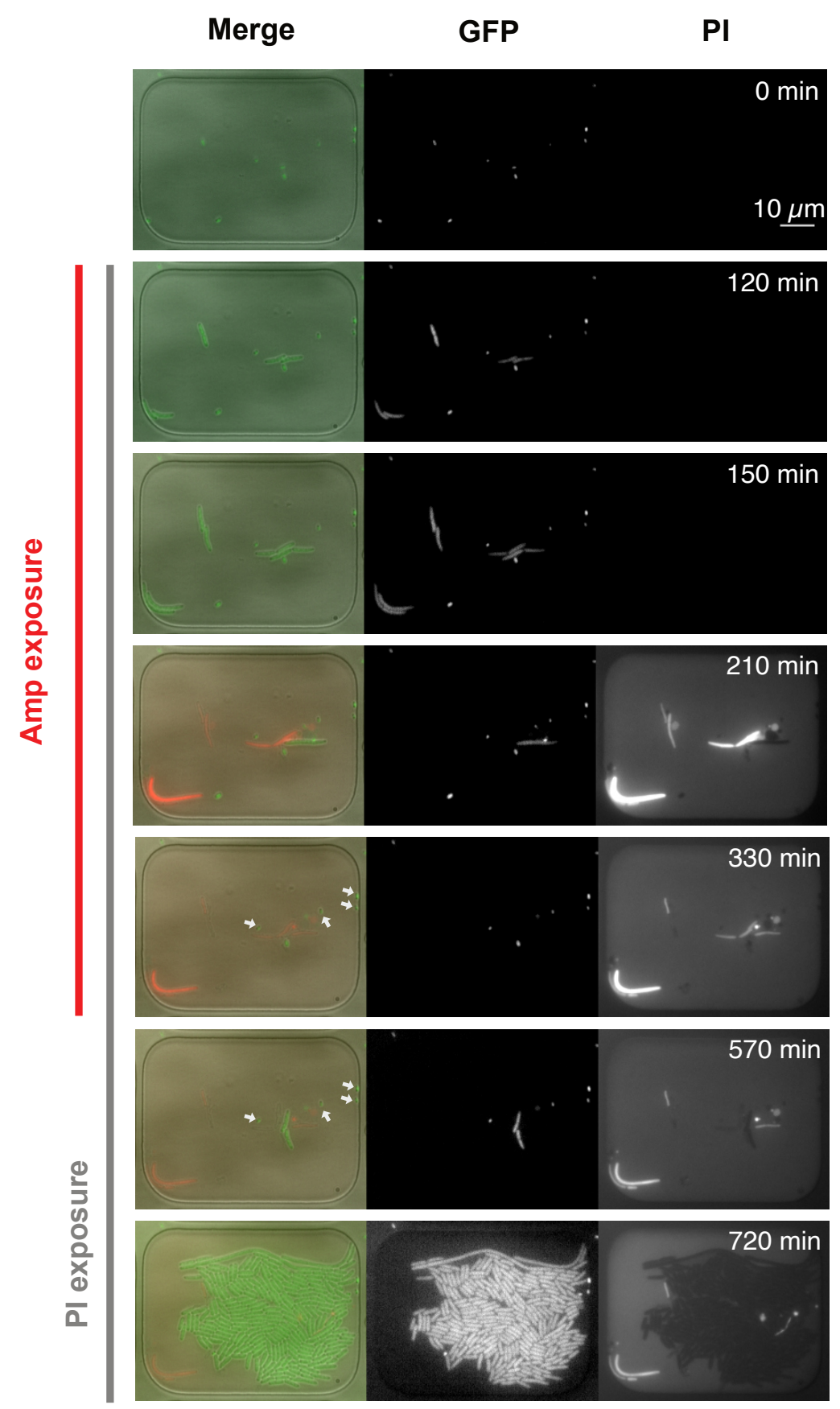

Figure S5. Viability check of the non-regrowing dormant cells by PI staining. The MG1655/pUA66-P rplS- $g f p$ strain was used in this experiment. The cells sampled from the late stationary phase were observed in the MCMA microfluidic device with a $100 \times$ objective. PI was added to the flowing media from the time point for starting the Amp exposure. The right and middle image sequences show the images of the PI and GFP fluorescence, respectively. The left image sequence shows the merged images of the bright-field (grayscale), GFP (green), and PI (red) channels. The white arrows in the merged images at $330 \mathrm{~min}$ and $570 \mathrm{~min}$ indicate several dormant cells that did not regrow after the Amp exposure. We detected no fluorescence signals of PI in these cells, which suggests that they were viable. 


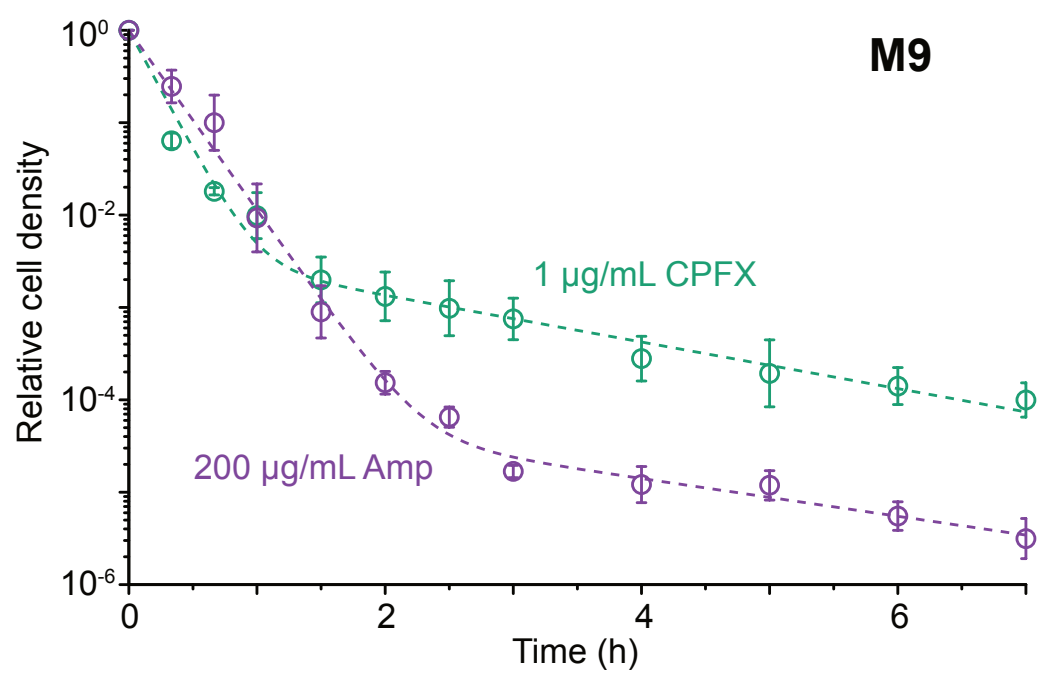

Figure S6. Population killing curve of the MF1 strain against CPFX. MF1 cells in the exponential phase were exposed to the M9 medium containing $1 \mathrm{\mu g} / \mathrm{ml}$ of CPFX. The cell density at different time points was evaluated by a limiting dilution method (green). The points represent the geometric means of the relative cell density to the values at the onset of the Amp exposure $(0 \mathrm{~min})$ among three replicate experiments. The error bars represent the standard errors of the logarithm of the relative cell density. The green dashed curve represents the fitting of a double exponential decay curve to the data: $N(t)=\left(1-4.32 \times 10^{-3}\right) \exp \left(-9.95 \times 10^{-2} t\right)+4.32 \times 10^{-3} \exp \left(-9.68 \times 10^{-3} t\right)$. The data points and the curve in purple are the results for the Amp exposure and the same as those of the "M9 Exponential phase" in Fig. S4. 